\title{
A DIRECT EULERIAN GRP SCHEME FOR COMPRESSIBLE FLUID FLOWS
}

\author{
MATANIA BEN-ARTZI, JIEQUAN LI AND GERALD WARNECKE
}

\begin{abstract}
A direct Eulerian generalized Riemann problem (GRP) scheme is derived for compressible fluid flows. Riemann invariants are introduced as the main ingredient to resolve the generalized Riemann problem (GRP) directly for the Eulerian formulation. The crucial auxiliary Lagrangian scheme in the original GRP scheme is not necessary in the present framework. The delicate sonic cases can be easily treated and the extension to multidimensional cases is straightforward.
\end{abstract}

Key words: The generalized Riemann problem (GRP) scheme, Eulerian method, Riemann invariants, characteristic coordinates.

\section{INTRODUCTION}

The generalized Riemann problem (GRP) scheme, an analytic extension of the Godunov scheme, was originally developed for compressible fluid dynamics $[1,4]$. It will be explained for the one dimensional system of an unsteady and inviscid flow in conservation form. The equations are

$$
\begin{gathered}
\frac{\partial U}{\partial t}+\frac{\partial F(U)}{\partial x}=0, \\
U=\left(\begin{array}{c}
\rho \\
\rho u \\
\rho\left(e+\frac{u^{2}}{2}\right)
\end{array}\right), \quad F(U)=\left(\begin{array}{c}
\rho u \\
\rho u^{2}+p \\
\rho u\left(e+\frac{u^{2}}{2}\right)+p u
\end{array}\right),
\end{gathered}
$$

where $\rho, u, e$ are density, velocity and internal energy, respectively, and $p=p(\rho, e)$ is the pressure. As is customary, we use the equally spaced grid points $x_{j}=j \Delta x$, the interface points $x_{j+1 / 2}=\left(x_{j}+x_{j+1}\right) / 2$ defining the cells $C_{j}=\left[x_{j-1 / 2}, x_{j+1 / 2}\right], j \in \mathbb{Z}$. Let $U_{j}^{n}$ be the average value of $U$ over the cell $C_{j}$ at time $t_{n}=n \Delta t$, and assume that the data at time $t=t_{n}$ are piesewise linear with a slope $\sigma_{j}^{n}$, i.e. on $C_{j}$ we have.

$$
U\left(x, t_{n}\right)=U_{j}^{n}+\sigma_{j}^{n}\left(x-x_{j}\right), \quad x \in\left(x_{j-1 / 2}, x_{j+1 / 2}\right) .
$$

Then the second order Godunov-type scheme for (1.1) takes the form

$$
U_{j}^{n+1}=U_{j}^{n}-\frac{\Delta t}{\Delta x}\left(F\left(U_{j+1 / 2}^{n+1 / 2}\right)-F\left(U_{j-1 / 2}^{n+1 / 2}\right)\right),
$$

where $U_{j+1 / 2}^{n+1 / 2}$ is the mid-point value or the value of $U$ at the cell interface $x=x_{j+1 / 2}$ averaged over the time interval $\left[t_{n}, t_{n+1}\right]$. The GRP scheme proceeds to derive the mid-point value $U_{j+1 / 2}^{n+1 / 2}$ analytically by resolving the generalized Riemann problem at each point $\left(x_{j+1 / 2}, t_{n}\right)$ 
with accuracy of second order. More specifically, the mid-point value $U_{j+1 / 2}^{n+1 / 2}$ is computed with the formulae

$$
U_{j+1 / 2}^{n+1 / 2}=U_{j+1 / 2}^{n}+\frac{\Delta t}{2}\left(\frac{\partial U}{\partial t}\right)_{j+1 / 2}^{n}, \quad U_{j+1 / 2}^{n}=R^{A}\left(0 ; U_{j+1 / 2,-}^{n}, U_{j+1 / 2,+}^{n}\right),
$$

where $R^{A}\left(\left(x-x_{j+1 / 2}\right) /\left(t-t_{n}\right) ; U_{j+1 / 2,-}^{n}, U_{j+1 / 2,+}^{n}\right)$ is the solution of the Riemann problem for (1.1) centered at $\left(x_{j+1 / 2}, t_{n}\right)$, and $U_{j+1 / 2,-}^{n}$ and $U_{j+1 / 2,+}^{n}$ are the limiting values of initial data $U\left(x, t_{n}\right)$ on both sides of $\left(x_{j+1 / 2}, t_{n}\right)$. With the Godunov scheme or the Riemann solution $U_{j+1 / 2}^{n}$ in mind, it is clear that only $(\partial U / \partial t)_{j+1 / 2}^{n}$ needs to be defined.

The GRP scheme was developed in $[1,4]$ and designed to deal with this problem. The main ingredient there is the analytic integration in time of the conservation laws (1.1). Two related versions, the Lagrangian and the Eulerian, are developed, and the Eulerian version is always derived by using the Lagrangian case. This approach has the advantage that the contact discontinuity in each local wave pattern is always fixed with speed zero and the rarefaction waves and/or shock waves are located on either side. The main issue is how to use characteristic coordinates in resolving centered rarefaction waves at the singularity point. However, the passage from the Lagrangian to the Eulerian version is sometimes quite delicate, particularly for sonic cases. An alternative approach by asymptotic analysis can be found in [5]. When just the Eulerian scheme is required, e.g., in the two-dimensional computation, it would be useful to have a direct derivation of the Eulerian scheme.

The purpose of this paper is to present a direct and simple derivation of the Eulerian generalized Riemann problem (GRP) scheme for compressible fluid flows. We indicate how to get the integration in time of the conservation laws (1.1) more directly and simply. Our approach is to apply Riemann invariants in order to resolve the singularity at the jump discontinuity. The new point enables us to get rid of the auxiliary Lagrangian scheme and has already been successfully applied to the shallow water equations with bottom topography [13]. The extension of this scheme to multidimensional cases is straightforward.

To be more precise, the main feature of the GRP scheme is the resolution of centered rarefaction waves. We first observe the following property of the Riemann invariants; they are constant throughout an isentropic rarefaction wave. This property implies that they are still regular inside the nonisentropic rarefaction wave occurring in the generalized Riemann problem, even though the derivatives of the flow variables $u, p$ and $\rho$ become singular at the initial discontinuity. Furthermore, the entropy is invariant along a streamline. When characteristic coordinates are used, the entropy equation is decoupled from the continuity and momentum equations so that we are able to solve it first. Then we are left with the Riemann invariants for the remaining two equations. Next we observe that the flow variables $u$ and $p$ are continuous across the contact discontinuity in the intermediate region so that we can first treat the directional derivatives of $u$ and $p$ and then proceed to calculate the derivatives of the density $\rho$ regardless of the location of the contact discontinuity. In addition, in the sonic case, one of the characteristic curves inside the rarefaction wave is tangential to the $t$-axis. This property enables us to apply the information already obtained for the rarefaction wave in order to compute the time derivatives of all flow variables. We recall that in the original GRP scheme [1], the sonic case is more delicate due to the nature of the transformation from the Lagrangian to the Eulerian framework. 
For the shock wave side, we just use the usual approach in order to resolve the discontinuity $[1,22]$. Thus we can obtain the instantaneous values of time derivatives in (1.4), simply through solving a linear algebraic system containing two equations in terms of material derivatives of $u$ and $p$. Therefore, this GRP scheme for (1.1), roughly speaking, consists of two steps: (i) Solving the Riemann problem at the discontinuity. (ii) Solving a linear system of two algebraic equations, where the coefficients only depend on the Riemann solution and the treatment of the GRP. In particular, the multidimensional extension is very simple. To summarize, the present approach has the following advantage over the original scheme [1]. (i) The transformation from the Lagrangian scheme is not necessary. (ii) We do not need to treat the sonic cases in a complicated way. (iii) The extension to the multidimensional cases is straightforward.

This paper is organized as follows. In Section 2 we first present some preliminaries and notations, including some basic relations among the flow variables and Riemann invariants. The resolution of rarefaction waves is treated in Section 3 and shocks are treated in Section 4. We conclude the solution of the generalized Riemann problem in Section 5 and the acoustic case in Section 6. The two dimensional extension is discussed in Section 7. It is the straightforward combination of our GRP scheme and the Strang splitting method. We outline the implementation of the GRP scheme in Section 8 and various standard 1-D and 2-D numerical test cases are presented in Section 9.

\section{Preliminaries and Notations}

In this section we present some preliminaries for the resolution of the generalized Riemann problem, particularly for rarefaction waves. Then we summarize the notations we use in the present paper for the reader's easy reference.

As is well-known [7], the system of Euler equations (1.1) takes the following form equivalently for smooth flows,

$$
\frac{D \rho}{D t}+\rho \frac{\partial u}{\partial x}=0, \quad \rho \frac{D u}{D t}+\frac{\partial p}{\partial x}=0, \quad \frac{D S}{D t}=0,
$$

where $D / D t=\partial / \partial t+u \partial / \partial x$ is the material derivative, and the entropy $S$ is related to the other variables through the second law of thermodynamics

$$
d e=T d S+\frac{p}{\rho^{2}} d \rho
$$

and $T$ is the temperature. Regard $p$ as a function of $\rho$ and $S, p=p(\rho, S)$. Then the local sound speed $c$ is defined as

$$
c^{2}=\frac{\partial p(\rho, S)}{\partial \rho} .
$$

Thus the first or third equation of (2.1) can be replaced equivalently by

$$
\frac{D p}{D t}+\rho c^{2} \frac{\partial u}{\partial x}=0
$$

Observe that the entropy $S$ is constant along a streamline. As the entropy is fixed, the continuity and momentum equations in (2.1) have the well-known feature of strictly hyperbolic conservation laws of two equations that Riemann invariants exist, see [18]. Therefore 
let us introduce the Riemann invariants $\phi$ and $\psi$,

$$
\phi=u-\int^{\rho} \frac{c(\omega, S)}{\omega} d \omega, \quad \psi=u+\int^{\rho} \frac{c(\omega, S)}{\omega} d \omega
$$

which play a pivotal role in the present study. Note that the entropy variable $S$ is automatically a Riemann invariant associated with $u-c$ or $u+c$. In terms of total differentials we can write, with all thermodynamic variables considered as functions of $\rho$ and $S$,

$$
d \psi=\frac{c}{\rho} d \rho+\frac{\partial \psi}{\partial S} d S+d u=\frac{1}{\rho c} d p+d u+K(\rho, S) d S
$$

where, since $\frac{\partial \psi}{\partial S}=\int^{\rho} \frac{1}{\omega} \cdot \frac{\partial c(\omega, S)}{\partial S} d \omega$, we have

$$
K(\rho, S)=-\frac{1}{\rho c} \cdot \frac{\partial p}{\partial S}+\int^{\rho} \frac{1}{\omega} \cdot \frac{\partial c(\omega, S)}{\partial S} d \omega .
$$

Recall [4, Eq. (4.67)] that along the characteristic $C_{+}: x^{\prime}(t)=u+c$ we have $\frac{1}{\rho c} d p+d u=0$, so that in this direction we get

$$
d \psi=K(\rho, S) d S .
$$

Observe that this can be further simplified if we note that, by $\partial S / \partial t+u \partial S / \partial x=0$, we have (along $C_{+}$),

$$
d S=c \frac{\partial S}{\partial x} d t
$$

Similarly, since $\frac{\partial \phi}{\partial S}=-\int^{\rho} \frac{1}{\omega} \cdot \frac{\partial c(\omega, S)}{\partial S} d \omega$, we have

$$
d \phi=d u-\frac{1}{\rho c} d p-K(\rho, S) d S,
$$

and, along $C_{-}: x^{\prime}(t)=u-c$,

$$
d \phi=-K(\rho, S) d S, \quad \text { and } \quad d S=-c \frac{\partial S}{\partial x} d t .
$$

In particular, in the important case of polytropic gases, we have

$$
p=(\gamma-1) \rho e, \quad \gamma>1,
$$

where $e$ is a function of $S$ alone. Then the Riemann invariants are

$$
\phi=u-\frac{2 c}{\gamma-1}, \quad \psi=u+\frac{2 c}{\gamma-1},
$$

where $c^{2}=\gamma p / \rho$. It follows that

$$
2 c \frac{\partial c}{\partial S}=\frac{\gamma}{\rho} \frac{\partial p}{\partial S}, \quad \text { and } \quad \frac{\partial \psi}{\partial S}=\frac{2}{(\gamma-1)} \frac{\partial c}{\partial S}=\frac{\gamma}{(\gamma-1) \rho c} \frac{\partial p}{\partial S} .
$$

In this case, by (2.7), we obtain

$$
K(\rho, S)=\frac{1}{(\gamma-1) \rho c} \frac{\partial p}{\partial S}=\frac{T}{c} .
$$

In view of (2.13), we have

$$
d \phi=d u-\frac{\gamma}{(\gamma-1) \rho c} d p+\frac{c}{(\gamma-1) \rho} d \rho, \quad d \psi=d u+\frac{\gamma}{(\gamma-1) \rho c} d p-\frac{c}{(\gamma-1) \rho} d \rho
$$




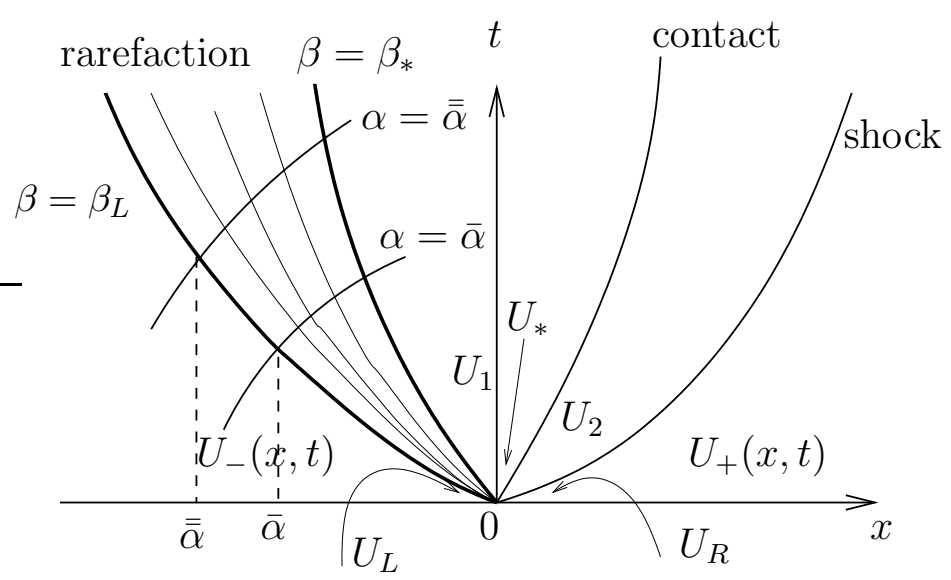

(a) Wave pattern for the GRP. The initial data $U_{0}(x)=U_{L}+$ $x U_{L}^{\prime}$ for $x<0$ and $U_{0}(x)=U_{R}+x U_{R}^{\prime}$ for $x>0$.

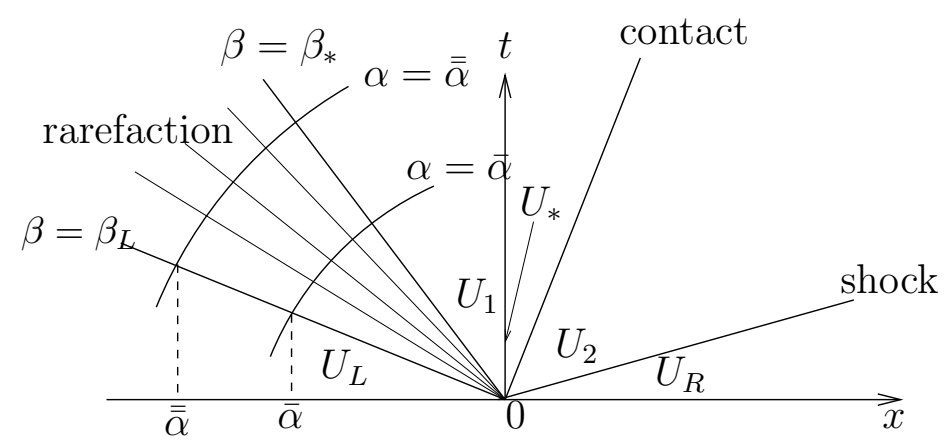

(b) Wave pattern for the associated Riemann problem

FiguRE 2.1. Typical wave configuration.

Also we note, combining (2.2) and (2.12),

$$
T d S=\frac{d p}{(\gamma-1) \rho}-\frac{c^{2}}{(\gamma-1) \rho} d \rho
$$

The GRP scheme assumes piecewise linear data for the flow variables. This leads to the generalized Riemann problem for (1.1) subject to the initial data

$$
U(x, 0)= \begin{cases}U_{L}+x U_{L}^{\prime}, & x<0, \\ U_{R}+x U_{R}^{\prime}, & x<0,\end{cases}
$$

where $U_{L}, U_{R}, U_{L}^{\prime}$ and $U_{R}^{\prime}$ are constant vectors. The initial structure of the solution $U(x, t)$ to $(1.1)$ and $(2.18)$ is determined by the associated Riemann solution, denoted by $R^{A}\left(x / t ; U_{L}, U_{R}\right)$, and

$$
\lim _{t \rightarrow 0} U(\lambda t, t)=R^{A}\left(\lambda ; U_{L}, U_{R}\right), \quad \lambda=x / t .
$$

The local wave configuration is usually piecewise smooth and consists of rarefaction waves, shocks and contact discontinuities, as the schematic description in Figure 2.1. We refer to $[7,4]$ for more details. The rarefaction wave as a part of the solution $R^{A}\left(x / t ; U_{L}, U_{R}\right)$, is referred to as the associated rarefaction wave. 
The flow is isentropic for the associated rarefaction waves. So $\psi$ (resp. $\phi$ ) and $S$ are constant inside the rarefaction wave associated with $u-c$ (resp. $u+c$ ) and their derivatives vanish. As the general (curved) rarefaction waves are considered, the initial data (2.18) can be regarded as a perturbation of the Riemann initial data $U_{L}, U_{R}$. We still expect $\psi$ (resp. $\phi)$ and $S$ to be regular inside the $(u-c)$-rarefaction wave (resp. $(u+c)$-rarefaction wave) at the singularity. As a key ingredient in this paper, we use the Riemann invariants to resolve the rarefaction waves at the singularity point.

Now we consider the wave configuration in Figure 2.1, a rarefaction wave moves to the left and a shock moves to the right. The intermediate region is separated by a contact discontinuity. The intermediate states in the two subregions are denoted by $U_{1}$ and $U_{2}$, respectively. Note that the pressure $p$ and velocity $u$ are continuous, $p_{1}=p_{2}, u_{1}=u_{2}$, and only the density has a jump across the contact discontinuity $\rho_{1} \neq \rho_{2}$. Finally, we denote by $U_{*}$ the limiting state at $x=0$, as $t \rightarrow 0+$. Otherwise stated, it is the result of the Riemann solution of the associated problem at $x=0$, with states $U_{R}, U_{L}$.

In the following table, we list some notations we will use in this paper.

TABLE I: Basic notations

\begin{tabular}{ll}
\hline Symbols & Definitions \\
\hline$\rho,(u, v), p, S$ & density, velocity componets, pressure, entropy \\
$\phi, \psi$ & Riemann invariants \\
$Q_{L}, Q_{R}$ & $\lim Q(x, 0)$ as $x \rightarrow 0_{-}, x \rightarrow 0_{+}$ \\
$Q_{L}^{\prime}, Q_{R}^{\prime}$, & constant slopes $\frac{\partial Q}{\partial x}$ for $x<0, x>0$ \\
$R^{A}\left(\cdot ; Q_{L}, Q_{R}\right)$ & solution of the Riemann problem subject to data $Q_{L}, Q_{R}$ \\
$Q_{*}$ & $R^{A}\left(0 ; Q_{L}, Q_{R}\right)$ \\
$Q_{1}, Q_{2}$ & the value of $Q$ to the left, the right of contact discontinuity \\
$Q_{-}(x, t), Q_{+}(x, t)$ & the solution in the left, the right \\
$\left(\frac{\partial Q}{\partial t}\right)_{*}$ & $\frac{\partial Q}{\partial t}(x, t)$ at $x=0$ as $t \rightarrow 0_{+}$ \\
$D Q / D t$ & the material derivative of $U, \frac{\partial Q}{\partial t}+u \frac{\partial Q}{\partial x}$ \\
$(D Q / D t)_{*}$ & the limiting value of $D Q / D t$ at $x=0$ as $t \rightarrow 0_{+}$ \\
$u-c, u, u+c$ & three eigenvalues \\
$\beta, \alpha$ & two characteristic coordinates \\
$\sigma_{L}, \sigma_{R}$ & shock speed at time zero, corresponding to $u-c, u+c$ \\
$\mu^{2}=\frac{\gamma-1}{\gamma+1}$ & $\gamma>1$ the polytropic index, $\gamma=1.4$ for air \\
\hline
\end{tabular}




\section{The RESOlUtion OF CENTERED RAREFACTION WAVES}

As already pointed out, the important feature of the GRP scheme is the treatment of the resolution of centered rarefaction waves with characteristic coordinates. Our objective is to obtain the time derivatives of the flow variables at the singularity point $(0,0)$.

Consider the rarefaction wave associated with $u-c$ and denote by $U_{-}(x, t)\left(\operatorname{resp} . U_{1}(x, t)\right)$ the states (regions of smooth flows) ahead (resp. behind) the rarefaction wave, see Figure 2.1(a), where $U_{-}(x, t)$ is determined by the left initial data $U_{L}+U_{L}^{\prime} x$. Characteristic curves throughout the rarefaction wave are denoted by $\beta(x, t)=\beta$ and $\alpha(x, t)=\alpha, \beta \in\left[\beta_{L}, \beta_{*}\right]$, $-\infty \leq \alpha<0, \beta_{L}=u_{L}-c_{L}, \beta_{*}=u_{*}-c_{*}$. They are the integral curves of the following equations, respectively,

$$
\frac{d x}{d t}=u-c, \quad \frac{d x}{d t}=u+c .
$$

Here $\beta$ and $\alpha$ are denoted as follows: $\beta$ is the initial value of the slope $u-c$ at the singularity $(x, t)=(0,0)$, and $\alpha$ for the transversal characteristic curves is the $x$-coordinate of the intersection point with the leading $\beta$-curve, which may be properly normalized, see below for polytropic gases. Then the coordinates $(x, t)$ can be expressed as

$$
x=x(\alpha, \beta), \quad t=t(\alpha, \beta),
$$

which satisfy

$$
\frac{\partial x}{\partial \alpha}=(u-c) \frac{\partial t}{\partial \alpha}, \quad \frac{\partial x}{\partial \beta}=(u+c) \frac{\partial t}{\partial \beta},
$$

and the characteristic equations for $\psi$ in (2.8) and $S$ in (2.9) become

$$
\frac{\partial S}{\partial \beta}=\frac{\partial t}{\partial \beta} \cdot c \frac{\partial S}{\partial x}, \quad \frac{\partial \psi}{\partial \beta}=\frac{\partial t}{\partial \beta} \cdot K(\rho, S) \frac{\partial S}{\partial x} .
$$

Differentiating the first equation in (3.3) with respect to $\beta$, the second with respect to $\alpha$, and subtracting, we see that the function $t=t(\alpha, \beta)$ satisfies,

$$
2 c \frac{\partial^{2} t}{\partial \alpha \partial \beta}=-\frac{\partial(u+c)}{\partial \alpha} \cdot \frac{\partial t}{\partial \beta}+\frac{\partial(u-c)}{\partial \beta} \cdot \frac{\partial t}{\partial \alpha} .
$$

As pointed out in Section 2, the initial structure of (1.1) and (2.18) is determined by the associated Riemann problem. So the rarefaction wave in Figure 2.1 (a) is asymptotically the same as the associated rarefaction wave $R^{A}$ in Figure 2.1(b) at the origin. The latter is expressed by using

$$
x / t=u-c, \quad \psi=\text { const }=\psi_{L}, \quad S=S_{L} .
$$

Note that the flow is isentropic throughout this associated rarefaction wave and recall (2.5) for the definition of $\psi$. Then it is reasonable to denote

$$
f_{L}(c):=\int^{\rho} c\left(\omega, S_{L}\right) \omega^{-1} d \omega+c
$$

which is invertible. Note that in view of (2.5), (3.6), we have

$$
\psi \equiv \psi_{L}=f_{L}(c)+x / t
$$

throughout the rarefaction wave of the associated Riemann problem. Hence, we obtain

$$
c=f_{L}^{-1}\left(\psi_{L}-x / t\right) .
$$


Therefore we get the characteristic coordinates for this associated rarefaction wave as follows: $\beta=x / t$ and $\alpha(x, t)=\bar{\alpha}$ is the integral curve

$$
\frac{d x}{d t}=u+c=x / t+2 f_{L}^{-1}\left(\psi_{L}-x / t\right)
$$

subject to the initial condition $x\left(t=\bar{\alpha} / \beta_{L}\right)=\bar{\alpha}$. Correspondingly, we denote $x$ and $t$ as functions of $\alpha$ and $\beta$,

$$
x=x_{\text {ass }}(\alpha, \beta), \quad t=t_{\text {ass }}(\alpha, \beta) .
$$

They are the leading terms (in powers of $\alpha$ ) of the transformation (3.2), as $\alpha \rightarrow 0$,

$$
x(\alpha, \beta)=x_{a s s}(\alpha, \beta)+O\left(\alpha^{2}\right), \quad t(\alpha, \beta)=t_{\text {ass }}(\alpha, \beta)+O\left(\alpha^{2}\right) .
$$

In particular, for the general rarefaction wave, see Figure 2.1(a), we have

$$
\frac{\partial(u-c)}{\partial \beta}(0, \beta)=1, \quad \frac{\partial t}{\partial \alpha}(0, \beta)=\frac{\partial t_{\text {ass }}}{\partial \alpha}(0, \beta), \quad \frac{\partial t}{\partial \beta}(0, \beta) \equiv 0, \quad \beta_{L} \leq \beta \leq \beta_{*} .
$$

For the case of polytropic gases, it follows from $(2.13)$ that $f_{L}(c)=\mu^{-2} c$, where $\mu^{2}=\frac{\gamma-1}{\gamma+1}$ so that throughout the rarefaction wave, we have

$$
u=\mu^{2} \psi_{L}+\left(1-\mu^{2}\right) x / t, \quad c=\mu^{2}\left(\psi_{L}-x / t\right) .
$$

The corresponding characteristic curves are

$$
\beta(x, t)=x / t, \quad \alpha(x, t)=t\left(\psi_{L}-x / t\right)^{1 /\left(2 \mu^{2}\right)} \cdot\left(c_{L} / \mu^{2}\right)^{-\frac{1}{2 \mu^{2}}} \cdot \beta_{L} .
$$

Denote $\alpha^{\prime}=\alpha \cdot\left(c_{L} / \mu^{2}\right)^{\frac{1}{2 \mu^{2}}} / \beta_{L}$. Then we have

$$
\beta(x, t)=x / t, \quad \alpha^{\prime}(x, t)=t\left(\psi_{L}-x / t\right)^{1 /\left(2 \mu^{2}\right)} .
$$

We use $\left(\alpha^{\prime}, \beta\right)$ as the characteristic coordinates from now on, and replace $\alpha^{\prime}$ by $\alpha$. Therefore, for the polytropic gases, we have

$$
t_{a s s}(\alpha, \beta)=\frac{\alpha}{\left(\psi_{L}-\beta\right)^{1 /\left(2 \mu^{2}\right)}}, \quad x_{a s s}(\alpha, \beta)=\frac{\alpha \beta}{\left(\psi_{L}-\beta\right)^{1 /\left(2 \mu^{2}\right)}} .
$$

The total derivatives $D u / D t$ and $D p / D t$ are functions of $\alpha, \beta$ throughout the rarefaction wave. A key ingredient in the resolution of the centered rarefaction wave (and, in fact, the GRP in general) is the fact that their limiting values, as $\alpha \rightarrow 0$, satisfy a simple linear relation, as expressed in the following lemma.

Lemma 3.1. The limiting values $(D u / D t)(0, \beta)$ and $(D p / D t)(0, \beta)$ satisfy the linear relation

$$
a_{L} \frac{D u}{D t}(0, \beta)+b_{L} \frac{D p}{D t}(0, \beta)=d_{L}(\beta),
$$

for all $\beta_{L} \leq \beta \leq \beta_{*}$, where

$$
\left(a_{L}, b_{L}\right)=\left(1, \frac{1}{\rho(0, \beta) c(0, \beta)}\right)
$$


and $d_{L}=d_{L}(\beta)$ is a function just depending on the initial data $U_{L}, U_{L}^{\prime}$, and the Riemann solution $R^{A}\left(x / t, ; U_{L}, U_{R}\right)$. For polytropic gases, $d_{L}$ is

$d_{L}=\left[\frac{1+\mu^{2}}{1+2 \mu^{2}}\left(\frac{c(0, \beta)}{c_{L}}\right)^{1 /\left(2 \mu^{2}\right)}+\frac{\mu^{2}}{1+2 \mu^{2}}\left(\frac{c(0, \beta)}{c_{L}}\right)^{\left(1+\mu^{2}\right) / \mu^{2}}\right] T_{L} S_{L}^{\prime}-c_{L}\left(\frac{c(0, \beta)}{c_{L}}\right)^{1 /\left(2 \mu^{2}\right)} \psi_{L}^{\prime}$.

Note that the limiting values $\rho(0, \beta), c(0, \beta)$ are obtained from the solution to the associated Riemann problem. Also, $T_{L} S_{L}^{\prime}, \psi_{L}^{\prime}$ are given by the formula (2.17) and (2.16), respectively.

Proof. The equation for $\psi$ in (2.6) and the equation for $S$ in (2.1) yield

$$
\frac{D u}{D t}+\frac{1}{\rho c} \frac{D p}{D t}=\frac{D \psi}{D t}
$$

So we only need to compute $D \psi / D t$ at $(0, \beta)$. From $(2.8)$ we have

$$
\frac{D \psi}{D t}=c K(\rho, S) \frac{\partial S}{\partial x}-c \frac{\partial \psi}{\partial x} \text {. }
$$

Denote

$$
A(\alpha, \beta):=c K(\rho, S) \cdot \frac{\partial S}{\partial x}(\alpha, \beta)
$$

It follows that we just need to compute $A(0, \beta)$ and $c(0, \beta) \frac{\partial \psi}{\partial x}(0, \beta)$ separately.

(i) The computation of $A(0, \beta)$. Note that $\partial S / \partial x$ is regarded as a function of $\alpha$ and $\beta$ although the derivative with respect to $x$ is involved. Since it vanishes identically in the case of the associated Riemann solution, it is a regular function of $\alpha$ and $\beta$. Then the characteristic equation for $S$ in (3.4) implies

$$
\frac{\partial^{2} S}{\partial \alpha \partial \beta}(\alpha, \beta)=\frac{\partial^{2} t}{\partial \alpha \partial \beta} \cdot c \frac{\partial S}{\partial x}+\frac{\partial t}{\partial \beta} \frac{\partial}{\partial \alpha}\left(c \frac{\partial S}{\partial x}\right) .
$$

Setting $\alpha=0$ and using (3.5), (3.13), one obtains

$$
\frac{\partial}{\partial \beta}\left(\frac{\partial S}{\partial \alpha}(0, \beta)\right)=\frac{1}{2 c(0, \beta)} \cdot \frac{\partial t}{\partial \alpha}(0, \beta) \cdot c(0, \beta) \cdot \frac{\partial S}{\partial x}(0, \beta)
$$

Thus with the fact that

$$
\frac{\partial S}{\partial \alpha}=\frac{\partial t}{\partial \alpha}\left(\frac{\partial S}{\partial t}+(u-c) \frac{\partial S}{\partial x}\right)=-c \frac{\partial t}{\partial \alpha} \frac{\partial S}{\partial x}
$$

we arrive at

$$
\frac{\partial}{\partial \beta}\left(\frac{\partial S}{\partial \alpha}(0, \beta)\right)=-\frac{1}{2 c} \frac{\partial S}{\partial \alpha}(0, \beta) .
$$

Integrating this equation from $\beta_{L}$ to $\beta$ yields

$$
\frac{\partial S(0, \beta)}{\partial \alpha}=\frac{\partial S\left(0, \beta_{L}\right)}{\partial \alpha} \exp \left(-\int_{\beta_{L}}^{\beta} \frac{1}{2 c(0, \xi)} d \xi\right)
$$

It follows, by using (3.26), that

$$
c \frac{\partial S}{\partial x}(0, \beta)=\left(\frac{\partial t_{a s s}}{\partial \alpha}\right)^{-1}(0, \beta)\left(\frac{\partial t_{a s s}}{\partial \alpha}\right)\left(0, \beta_{L}\right) \cdot c_{L} S_{L}^{\prime} \exp \left(-\int_{\beta_{L}}^{\beta} \frac{1}{2 c(0, \xi)} d \xi\right) .
$$


That is, we get

$$
A(0, \beta)=K\left(\rho(0, \beta), S_{L}\right) \cdot\left(\frac{\partial t_{\text {ass }}}{\partial \alpha}\right)^{-1}(0, \beta)\left(\frac{\partial t_{\text {ass }}}{\partial \alpha}\right)\left(0, \beta_{L}\right) \cdot c_{L} S_{L}^{\prime} \exp \left(-\int_{\beta_{L}}^{\beta} \frac{1}{2 c(0, \xi)} d \xi\right) .
$$

Particularly, for the polytropic gases, we have, by using (3.14) and (3.17),

$$
\frac{\partial t_{a s s}(0, \beta)}{\partial \alpha}=\frac{1}{\left(\psi_{L}-\beta\right)^{\frac{1}{2 \mu^{2}}}}=\frac{1}{\left(c / \mu^{2}\right)^{\frac{1}{2 \mu^{2}}}}, \quad \exp \left(-\int_{\beta_{L}}^{\beta} \frac{1}{2 c(0, \xi)} d \xi\right)=\left(\frac{c}{c_{L}}\right)^{\frac{1}{2 \mu^{2}}} .
$$

We use (2.2) and (2.12) to get $T / T_{L}=c^{2} / c_{L}^{2}$. Therefore, we conclude, by recalling (2.15), for the case of polytropic gases,

$$
A(0, \beta)=\left(\frac{c}{c_{L}}\right)^{\left(1+\mu^{2}\right) / \mu^{2}} T_{L} S_{L}^{\prime}
$$

where $T_{L} S_{L}^{\prime}$ is given by $(2.17)$.

(ii) The computation of $c(0, \beta) \cdot \frac{\partial \psi}{\partial x}(0, \beta)$. First we observe, using (2.8),

$$
\begin{aligned}
\frac{\partial \psi}{\partial \alpha}(0, \beta) & =\frac{\partial t}{\partial \alpha}(0, \beta)\left[\frac{\partial \psi}{\partial t}+(u-c) \frac{\partial \psi}{\partial x}\right](0, \beta) \\
& =\frac{\partial t}{\partial \alpha}(0, \beta)\left[\frac{\partial \psi}{\partial t}+(u+c) \frac{\partial \psi}{\partial x}-2 c \frac{\partial \psi}{\partial x}\right](0, \beta) \\
& =\frac{\partial t}{\partial \alpha}(0, \beta)\left[A(0, \beta)-2 c(0, \beta) \cdot \frac{\partial \psi}{\partial x}(0, \beta)\right] .
\end{aligned}
$$

That is

$$
c(0, \beta) \frac{\partial \psi}{\partial x}(0, \beta)=-\frac{1}{2}\left[\left(\frac{\partial t_{\text {ass }}}{\partial \alpha}\right)^{-1}(0, \beta) \cdot \frac{\partial \psi}{\partial \alpha}(0, \beta)-A(0, \beta)\right] .
$$

Note that $A(0, \beta)$, as function of $\beta$, is already known in (3.30). Therefore we are left with the calculation of $(\partial \psi / \partial \alpha)(0, \beta)$. The characteristic equation for $\psi$ in (3.4) gives

$$
\frac{\partial^{2} \psi}{\partial \alpha \partial \beta}=\frac{\partial^{2} t}{\partial \alpha \partial \beta} \cdot A(\alpha, \beta)+\frac{\partial t}{\partial \beta} \frac{\partial A(\alpha, \beta)}{\partial \alpha} .
$$

Setting $\alpha=0$ and recalling (3.5), (3.13), we obtain

$$
\frac{\partial}{\partial \beta}\left(\frac{\partial \psi}{\partial \alpha}(0, \beta)\right)=\frac{1}{2 c(0, \beta)} \cdot \frac{\partial t_{\text {ass }}}{\partial \alpha}(0, \beta) \cdot A(0, \beta) .
$$

The integration from $\beta_{L}$ to $\beta$ gives,

$$
\frac{\partial \psi}{\partial \alpha}(0, \beta)=\frac{\partial \psi}{\partial \alpha}\left(0, \beta_{L}\right)+\int_{\beta_{L}}^{\beta} \frac{1}{2 c(0, \xi)} \cdot \frac{\partial t_{a s s}}{\partial \alpha}(0, \xi) \cdot A(0, \xi) d \xi .
$$

where the initial data $(\partial \psi / \partial \alpha)\left(0, \beta_{L}\right)$ is obtained from (3.33) by setting $\beta=\beta_{L}$ and $(\partial \psi / \partial x)\left(0, \beta_{L}\right)=\psi_{L}^{\prime}$. 
For the polytropic gases, by using (3.14), (3.17) and (3.32) and noting $T / T_{L}=c^{2} / c_{L}^{2}$, we obtain

$$
\frac{\partial \psi}{\partial \alpha}(0, \beta)=\frac{\partial \psi}{\partial \alpha}\left(0, \beta_{L}\right)-\frac{2 B}{1+2 \mu^{2}}\left(c^{\left(1+2 \mu^{2}\right) /\left(2 \mu^{2}\right)}-c_{L}^{\left(1+2 \mu^{2}\right) /\left(2 \mu^{2}\right)}\right) .
$$

where

$$
B=\frac{1}{2}\left(\mu^{2}\right)^{1 /\left(2 \mu^{2}\right)} c_{L}^{-\left(\mu^{2}+1\right) / \mu^{2}} T_{L} S_{L}^{\prime}
$$

Inserting (3.32) and (3.38) into (3.22), we get the right-hand side of (3.21), as given by (3.20) (for the polytropic gases).

\section{The RESOlution OF SHOCKS}

In this section, we follow the idea of $[22]$ in order to resolve the shock at the origin. Our objective is to get an equation, which is analogous to (3.18). In other words, we look for another linear relation for the time derivatives of $u$ and $p$, which can be used to obtain the limiting values $(D u / D t)_{*}$ and $(D p / D t)_{*}$ at $(x, t)=\left(0,0_{+}\right)$(see Theorem 5.1).

Let $x=x(t)$ be the shock trajectory which is associated with the $u+c$ characteristic family and assume that it propagates with the speed $\sigma=x^{\prime}(t)>0$ to the right, see Figure 2.1(a). We use $\bar{Q}(t)=Q(x(t)+0, t)$ and $Q(t)=Q(x(t)-0, t)$ to denote the preshock and post shock values of $Q$, respectively. Along this shock, the $(p, u)$-Rankine-Hugoniot relation is written in the form,

$$
u=\bar{u}+\Phi(p ; \bar{p}, \bar{\rho}),
$$

and the $(\rho, p)$-Rankine-Hugoniot relation takes the form,

$$
\rho=H(p ; \bar{p}, \bar{\rho}) \text {. }
$$

The shock velocity is given by

$$
\sigma=\frac{\rho u-\overline{\rho u}}{\rho-\bar{\rho}} .
$$

We take the directional derivative along the shock trajectory $x=x(t)$ to get,

$$
\left(\frac{\partial}{\partial t}+\sigma \frac{\partial}{\partial x}\right) \Gamma=0
$$

where either $\Gamma=u-\bar{u}-\Phi(p ; \bar{p}, \bar{\rho})$ or $\Gamma=\rho-H(p ; \bar{p}, \bar{\rho})$. The continuity property of solutions adjacent to the shock front implies that we can replace the time derivatives of $\bar{U}$ by the $x$ derivatives in the preshock region, and similarly we replace the $x$-derivatives of $U$ by the time derivatives in the post shock region, for which (2.1), (2.4) are used. In the setup of Figure 2.1(a), $\bar{U}$ is given by $U_{+}(x, t)$ and $U$ is given by $U_{2}$. Note that the variables $u$ and $p$ are continuous across the contact discontinuity with the speed $u$ and thereby the total derivatives $D u / D t$ and $D p / D t$ are also continuous in the intermediate region between the rarefaction wave and the shock. Therefore, by taking the limit $t \rightarrow 0_{+}$, we get,

$$
\frac{D u}{D t} \rightarrow\left(\frac{D u}{D t}\right)_{*}, \quad \frac{D p}{D t} \rightarrow\left(\frac{D p}{D t}\right)_{*}, \quad \frac{\partial \bar{U}}{\partial x} \rightarrow U_{R}^{\prime}
$$

and also

$$
(\rho, u, p) \rightarrow\left(\rho_{2 *}, u_{*}, p_{*}\right), \quad(\bar{\rho}, \bar{u}, \bar{p}) \rightarrow\left(\rho_{R}, u_{R}, p_{R}\right)
$$


Note again that $\rho$ undergoes a jump across the contact discontinuity. This is why we write $\rho_{2 *}$ in (4.6), which is the limiting value of the density between the contact discontinuity and the shock. The same thing applies to the limiting value of $c_{2 *}$.

Lemma 4.1. The limiting values $(D u / D t)_{*}$ and $(D p / D t)_{*}$ satisfy

$$
a_{R}\left(\frac{D u}{D t}\right)_{*}+b_{R}\left(\frac{D p}{D t}\right)_{*}=d_{R}
$$

where $a_{R}, b_{R}, d_{R}$ are constant, depending only on the right hand of initial data (2.18), $U_{R}$, $U_{R}^{\prime}$, and the solution $R^{A}\left(0 ; U_{L}, U_{R}\right)$ to the associated Riemann problem. They are given by the following expressions,

$$
a_{R}=1+\rho_{2 *} \cdot\left(\sigma-u_{*}\right) \cdot \Phi_{1}, \quad b_{R}=-\left[\frac{1}{\rho_{2 *} \cdot c_{2 *}^{2}} \cdot\left(\sigma-u_{*}\right)+\Phi_{1}\right],
$$

$$
d_{R}=L_{p}^{R} \cdot p_{R}^{\prime}+L_{u}^{R} \cdot u_{R}^{\prime}+L_{\rho}^{R} \cdot \rho_{R}^{\prime}
$$

and

$$
\begin{aligned}
& L_{p}^{R}=-\frac{1}{\rho_{R}}+\left(\sigma-u_{R}\right) \cdot \Phi_{2}, \\
& L_{u}^{R}=\sigma-u_{R}-\rho_{R} \cdot c_{R}^{2} \cdot \Phi_{2}-\rho_{R} \cdot \Phi_{3}, \\
& L_{\rho}^{R}=\left(\sigma-u_{R}\right) \cdot \Phi_{3} .
\end{aligned}
$$

Here $\Phi_{i}, i=1,2,3$, are

$$
\Phi_{1}=\frac{\partial \Phi}{\partial p}\left(p_{*} ; p_{R}, \rho_{R}\right), \quad \Phi_{2}=\frac{\partial \Phi}{\partial \bar{p}}\left(p_{*} ; p_{R}, \rho_{R}\right), \quad \Phi_{3}=\frac{\partial \Phi}{\partial \bar{\rho}}\left(p_{*} ; p_{R}, \rho_{R}\right) .
$$

Proof. We follow the differentiation (4.4) for $\Gamma=u-\bar{u}-\Phi(p, \bar{p}, \bar{\rho})$ to get

$$
\begin{aligned}
\frac{\partial u}{\partial t}+\sigma \frac{\partial u}{\partial x}= & \frac{\partial \bar{u}}{\partial t}+\sigma \frac{\partial \bar{u}}{\partial x}+\frac{\partial \Phi}{\partial p} \cdot\left(\frac{\partial p}{\partial t}+\sigma \frac{\partial p}{\partial x}\right) \\
& +\frac{\partial \Phi}{\partial \bar{p}} \cdot\left(\frac{\partial \bar{p}}{\partial t}+\sigma \frac{\partial \bar{p}}{\partial x}\right)+\frac{\partial \Phi}{\partial \bar{\rho}} \cdot\left(\frac{\partial \bar{\rho}}{\partial t}+\sigma \frac{\partial \bar{\rho}}{\partial x}\right) .
\end{aligned}
$$

Using (2.1) and (2.4), we have

$$
\begin{gathered}
\frac{\partial u}{\partial t}+\sigma \frac{\partial u}{\partial x}=\frac{D u}{D t}-\frac{1}{\rho c^{2}}(\sigma-u) \frac{D p}{D t}, \\
\frac{\partial p}{\partial t}+\sigma \frac{\partial p}{\partial x}=\frac{D p}{D t}-\rho(\sigma-u) \frac{D u}{D t} .
\end{gathered}
$$

Then we use (2.1) and (2.4) again to replace the time derivatives of $\bar{p}, \bar{\rho}$ by the corresponding space derivatives and proceed to take the limit $t \rightarrow 0_{+}$for the resulting equation to finally obtain (4.7). 
Remark 4.2. ( $\gamma$-law case.) In the polytropic case, we have (see [14, Chapter 5] for the definition of $\Phi)$

$$
\begin{aligned}
& \Phi(p ; \bar{p}, \bar{\rho})=(p-\bar{p}) \sqrt{\frac{1-\mu^{2}}{\bar{\rho}\left(p+\mu^{2} \bar{p}\right)}}, \\
& \Phi_{1}=\frac{1}{2} \sqrt{\frac{1-\mu^{2}}{\rho_{R}\left(p_{*}+\mu^{2} p_{R}\right)} \cdot \frac{p_{*}+\left(1+2 \mu^{2}\right) p_{R}}{p_{*}+\mu^{2} p_{R}}}, \\
& \Phi_{2}=-\frac{1}{2} \sqrt{\frac{1-\mu^{2}}{\rho_{R}\left(p_{*}+\mu^{2} p_{R}\right)}} \cdot \frac{\left(2+\mu^{2}\right) p_{*}+\mu^{2} p_{R}}{p_{*}+\mu^{2} p_{R}}, \\
& \Phi_{3}=-\frac{p_{*}-p_{R}}{2 \rho_{R}} \sqrt{\frac{1-\mu^{2}}{\rho_{R}\left(p_{*}+\mu^{2} p_{R}\right)}} .
\end{aligned}
$$

\section{Time Derivative OF SOlutions at the Singularity}

In this section we use the results of Sections 3 and 4 in order to calculate the instantaneous value $(\partial U / \partial t)_{*}$. We assume the setup of Figure 2.1, i.e, the rarefaction wave moves to the left and the shock moves to the right, separated by a contact discontinuity with the speed $u$. Due to the continuity property of the pressure $p$ (resp. the velocity $u$ ), the total derivative $D p / D t$ (resp. $D u / D t$ ) is continuous across the contact discontinuity and thus the limiting values $(D p / D t)_{*}\left(\operatorname{resp} .(D u / D t)_{*}\right)$ are the same in the two subregions. Hence it is convenient to first calculate $(D u / D t)_{*},(D p / D t)_{*}$, and then turn to $(\partial u / \partial t)_{*},(\partial p / \partial t)_{*}$. The value $(\partial \rho / \partial t)_{*}$ then follows immediately. For this purpose, we summarize the results in Lemmas 3.1 and 4.1 to get the following theorem.

Theorem 5.1. (Nonsonic case.) Assume that the t-axis is not included in the rarefaction wave. Then the limiting values $(D u / D t)_{*}$ and $(D p / D t)_{*}$ are obtained by solving a pair of linear algebraic equations

$$
\begin{aligned}
& a_{L}\left(\frac{D u}{D t}\right)_{*}+b_{L}\left(\frac{D p}{D t}\right)_{*}=d_{L}, \\
& a_{R}\left(\frac{D u}{D t}\right)_{*}+b_{R}\left(\frac{D p}{D t}\right)_{*}=d_{R},
\end{aligned}
$$

where $a_{L}, a_{R}, b_{L}, b_{R}, d_{L}$ and $d_{R}$ are defined in Lemmas 3.1 and 4.1, and summarized for all cases in Appendix A, respectively. These coefficients depend only on the initial data (2.18) and the associated Riemann solution $R^{A}\left(0 ; U_{L}, U_{R}\right)$.

We now proceed to the basic step of the GRP solution (see (1.4), i.e, the calculation of $(\partial U / \partial t)_{*}$. 
Theorem 5.2. (Nonsonic case.) The limiting values of time derivatives $(\partial u / \partial t)_{*}$ and $(\partial p / \partial t)_{*}$ are calculated with the following formulae

$$
\begin{aligned}
& \left(\frac{\partial u}{\partial t}\right)_{*}=\left(\frac{D u}{D t}\right)_{*}+\frac{u_{*}}{\rho_{*} c_{*}^{2}}\left(\frac{D p}{D t}\right)_{*}, \\
& \left(\frac{\partial p}{\partial t}\right)_{*}=\left(\frac{D p}{D t}\right)_{*}+\rho_{*} u_{*}\left(\frac{D u}{D t}\right)_{*} .
\end{aligned}
$$

Proof. From (2.4) we get,

$$
\frac{\partial u}{\partial t}=\frac{D u}{D t}-u \frac{\partial u}{\partial x}=\frac{D u}{D t}+\frac{u}{\rho c^{2}} \frac{D p}{D t} .
$$

Then we get $(\partial u / \partial t)_{*}$ by taking the limit $t \rightarrow 0_{+}$. Similarly we can get the expression from $(2.1)$ for $(\partial p / \partial t)_{*}$ in $(5.2)$.

Remark 5.3. Note that in the setup of Figure 2.1, $\rho_{*}, c_{*}$ are those obtained behind the contact discontinuity (compare Eq. (4.6) and the paragraph after it).

When the $t$-axis $(x=0)$ is located inside a rarefaction fan, we have a sonic case, and Theorems 5.1 and 5.2 do not apply. However, since one of the characteristic curves becomes tangential to the $t$-axis, the situation becomes much simpler. Indeed, we have the following theorem.

Theorem 5.4. (Sonic case.) Assume that the $t$-axis is located inside the rarefaction wave associated with the $u-c$ characteristic family. Then we have

$$
\left(\frac{\partial u}{\partial t}\right)_{*}=d_{L}(0), \quad\left(\frac{\partial p}{\partial t}\right)_{*}=\rho_{*} u_{*} d_{L}(0),
$$

where $d_{L}(\beta)$ is defined in Lemma 3.1.

Proof. On one hand, using (2.6), we have

$$
\frac{\partial u}{\partial t}+\frac{1}{\rho c} \frac{\partial p}{\partial t}=\frac{\partial \psi}{\partial t}-K(\rho, S) \frac{\partial S}{\partial t}=\frac{\partial \psi}{\partial t}+u K(\rho, S) \frac{\partial S}{\partial x}
$$

Using (2.8) and (2.9), we proceed to get

$$
K(\rho, S) \frac{\partial S}{\partial x}=\frac{K(\rho, S)}{c}\left(\frac{\partial S}{\partial t}+(u+c) \frac{\partial S}{\partial x}\right)=\frac{1}{c}\left(\frac{\partial \psi}{\partial t}+(u+c) \frac{\partial \psi}{\partial x}\right) .
$$

Then from (5.5), we obtain

$$
\frac{\partial u}{\partial t}+\frac{1}{\rho c} \frac{\partial p}{\partial t}=\frac{u+c}{c} \frac{D \psi}{D t}
$$

With the results in (3.18) and (3.21), we conclude that $(D \psi / D t)_{*}=d_{L}(0)$. Then we get

$$
\left(\frac{\partial u}{\partial t}\right)_{*}+\frac{1}{\rho_{*} c_{*}}\left(\frac{\partial p}{\partial t}\right)_{*}=2 d_{L}(0) .
$$


On the other hand, using the fact that at the origin the $t$-axis is tangential to the characteristic curve defined by $u_{*}-c_{*}$ and using (2.11), we have

$$
\begin{aligned}
\left(\frac{\partial \phi}{\partial t}\right)_{*} & =\left(\frac{\partial \phi}{\partial t}\right)_{*}+\left(u_{*}-c_{*}\right)\left(\frac{\partial \phi}{\partial x}\right)_{*} \\
& =-K\left(\rho_{*}, S_{*}\right)\left[\left(\frac{\partial S}{\partial t}\right)_{*}+\left(u_{*}-c_{*}\right)\left(\frac{\partial S}{\partial x}\right)_{*}\right] \\
& =-K\left(\rho_{*}, S_{*}\right)\left(\frac{\partial S}{\partial t}\right)_{*}
\end{aligned}
$$

It follows that

$$
\left(\frac{\partial u}{\partial t}\right)_{*}-\frac{1}{\rho_{*} c_{*}}\left(\frac{\partial p}{\partial t}\right)_{*}=\left(\frac{\partial \phi}{\partial t}\right)_{*}+K\left(\rho_{*}, S_{*}\right)\left(\frac{\partial S}{\partial t}\right)_{*}=0 .
$$

where the formula (2.10) is used. Note that indeed (5.10) follows directly from the characteristic relation satisfied along u-c characteristics. We combine (5.8) and (5.10) to yield $(5.4)$.

Now we are left with the calculation of $(\partial \rho / \partial t)_{*}$. This calculation depends on whether the contact discontinuity propagates to the left or the right. In other words, we calculate $(\partial \rho / \partial t)_{*}$ in the left hand side if $u_{*}>0$; and in the right-hand side if $u_{*}<0$.

Theorem 5.5. (General Case.) The limiting value $(\partial \rho / \partial t)_{*}$ is calculated as follows.

(i) If $u_{*}>0$, it is obtained by the formula

$$
\left(\frac{\partial \rho}{\partial t}\right)_{*}=\frac{1}{c_{*}^{2}}\left(\left(\frac{\partial p}{\partial t}\right)_{*}+\frac{\partial p}{\partial S}\left(\rho_{*}, S_{*}\right) \cdot \frac{u_{*}}{c_{*} K\left(\rho_{*}, S_{*}\right)} A\left(0, \beta_{*}\right)\right),
$$

where $A\left(0, \beta_{*}\right)$ is given in (3.30).

(ii) If $u_{*}<0$, the limiting value $(\partial \rho / \partial t)_{*}$ is calculated by the formula

$$
g_{\rho}^{R}\left(\frac{\partial \rho}{\partial t}\right)_{*}+g_{p}^{R}\left(\frac{D p}{D t}\right)_{*}+g_{u}^{R}\left(\frac{D u}{D t}\right)_{*}=f_{R}
$$

where $g_{\rho}^{R}, g_{p}^{R}, g_{u}^{R}$ and $f_{R}$ are constant, depending on the initial data (2.18) in the right hand side and the Riemann solution $R^{A}\left(0 ; U_{L}, U_{R}\right)$. They are expressed in the following,

$$
g_{\rho}^{R}=1-\frac{\sigma}{u_{*}}, \quad g_{p}^{R}=\frac{\sigma}{c_{*}^{2} u_{*}}-H_{1}, \quad g_{u}^{R}=\rho_{*}\left(\sigma-u_{*}\right) \cdot H_{1},
$$

$$
f_{R}=\left(\sigma-u_{R}\right) \cdot H_{2} \cdot p_{R}^{\prime}+\left(\sigma-u_{R}\right) \cdot H_{3} \cdot \rho_{R}^{\prime}-\rho_{R} \cdot\left(H_{2} \cdot c_{R}^{2}+H_{3}\right) \cdot u_{R}^{\prime} .
$$

Here $\sigma$ is given in (4.3), and $H_{i}, i=1,2,3$, are

$$
H_{1}=\frac{\partial H}{\partial p}\left(p_{*} ; p_{R}, \rho_{R}\right), \quad H_{2}=\frac{\partial H}{\partial \bar{p}}\left(p_{*} ; p_{R}, \rho_{R}\right), \quad H_{3}=\frac{\partial H}{\partial \bar{\rho}}\left(p_{*} ; p_{R}, \rho_{R}\right) .
$$

Recall that $H$ is defined in (4.2). 
Proof. For the first case that $u_{*}>0$, we use the equation of state $p=p(\rho, S)$ and $\frac{\partial S}{\partial t}=-u \frac{\partial S}{\partial x}$ by the fact in $(2.1)$ to get

$$
\frac{\partial p}{\partial t}=c^{2} \frac{\partial \rho}{\partial t}+\frac{\partial p}{\partial S} \frac{\partial S}{\partial t}=c^{2} \frac{\partial \rho}{\partial t}-u \frac{\partial p}{\partial S} \frac{\partial S}{\partial x} .
$$

Then we use the definition of $A(\alpha, \beta)$ in (3.23) in order to obtain (5.11) after setting $(\alpha, \beta)=$ $\left(0, \beta_{*}\right)$.

For the second case that $u_{*}<0$, we follow the differentiation (4.4) for $\Gamma=\rho-\bar{\rho}-H(\rho ; \bar{p}, \bar{\rho})$, exactly as was done in the proof of Lemma 4.1.

Remark 5.6. ( $\gamma$-law case.) In the case of polytropic gases, we have the explicit formulae for (5.11) and $H_{i}, i=1,2,3$, in (5.14). Indeed, using (2.12), (2.15) and (3.32), (5.11) becomes,

$$
\left(\frac{\partial \rho}{\partial t}\right)_{*}=\frac{1}{c_{*}^{2}}\left(\left(\frac{\partial p}{\partial t}\right)_{*}+(\gamma-1) \rho_{*} u_{*}\left(\frac{c_{*}}{c_{L}}\right)^{\left(1+\mu^{2}\right) / \mu^{2}} T_{L} S_{L}^{\prime}\right) .
$$

The explicit formulae for $H$ and $H_{i}, i=1,2,3$, are (see [14, Chapter 5] for the definition of $H)$

$$
H(p ; \bar{p}, \bar{\rho})=\bar{\rho} \frac{p+\mu^{2} \bar{p}}{\bar{p}+\mu^{2} p}, \quad H_{1}=\frac{\rho_{R}\left(1-\mu^{4}\right) p_{R}}{\left(p_{R}+\mu^{2} p_{*}\right)^{2}}, \quad H_{2}=\frac{\rho_{R}\left(\mu^{4}-1\right) p_{*}}{\left(p_{R}+\mu^{2} p_{*}\right)^{2}}, \quad H_{3}=\frac{p_{*}+\mu^{2} p_{R}}{p_{R}+\mu^{2} p_{*}} .
$$

\section{Acoustic Case}

When $U_{L}=U_{R}$ and $U_{L}^{\prime} \neq U_{R}^{\prime}$, the acoustic case follows. Then only linear waves emanate from the origin. This scheme thus becomes simple and is stated in the following theorem.

Theorem 6.1. (Acoustic case.) When $U_{L}=U_{*}=U_{R}$ and $U_{L}^{\prime} \neq U_{R}^{\prime}$, we have the acoustic case. If $u_{*}-c_{*}<0$ and $u_{*}+c_{*}>0$, then $(\partial u / \partial t)_{*}$ and $(\partial p / \partial t)_{*}$ can be solved to be

$$
\begin{gathered}
\left(\frac{\partial u}{\partial t}\right)_{*}=-\frac{1}{2}\left[\left(u_{*}+c_{*}\right)\left(u_{L}^{\prime}+\frac{p_{L}^{\prime}}{\rho_{*} c_{*}}\right)+\left(u_{*}-c_{*}\right)\left(u_{R}^{\prime}-\frac{p_{R}^{\prime}}{\rho_{*} c_{*}}\right)\right], \\
\left(\frac{\partial p}{\partial t}\right)_{*}=-\frac{\rho_{*} c_{*}}{2}\left[\left(u_{*}+c_{*}\right)\left(u_{L}^{\prime}+\frac{p_{L}^{\prime}}{\rho_{*} c_{*}}\right)-\left(u_{*}-c_{*}\right)\left(u_{R}^{\prime}-\frac{p_{R}^{\prime}}{\rho_{*} c_{*}}\right)\right] .
\end{gathered}
$$

Then the quantity $(\partial \rho / \partial t)_{*}$ is calculated from the equation of state $p=p(\rho, S)$,

$$
\left(\frac{\partial \rho}{\partial t}\right)_{*}= \begin{cases}\frac{1}{c_{*}^{2}}\left[\left(\frac{\partial p}{\partial t}\right)_{*}+u_{*}\left(p_{L}^{\prime}-c_{*}^{2} \rho_{L}^{\prime}\right)\right], & \text { if } u_{L}=u_{*}=u_{R}>0, \\ \frac{1}{c_{*}^{2}}\left[\left(\frac{\partial p}{\partial t}\right)_{*}+u_{*}\left(p_{R}^{\prime}-c_{*}^{2} \rho_{R}^{\prime}\right)\right], & \text { if } u_{L}=u_{*} u_{R}<0 .\end{cases}
$$

Proof. First we consider the acoustic wave in the left. Denote by $U_{-}(x, t), U_{1}(x, t)$ the states in the left hand side and the right hand side of the $u-c$ characteristic curve emanating from 
the origin, respectively. See the setup in Figure 2.1. Since the solution is continuous across this characteristic curve, we take the differentiation along it for the variable $u$ and get

$$
\frac{\partial u_{-}}{\partial t}+(u-c) \frac{\partial u_{-}}{\partial x}=\frac{\partial u_{1}}{\partial t}+(u-c) \frac{\partial u_{1}}{\partial x} .
$$

Using (2.1) and (2.4), we have

$$
\frac{D u_{-}}{D t}-c \frac{\partial u_{-}}{\partial x}=\frac{\partial u_{1}}{\partial t}-\frac{u-c}{\rho c^{2}} \cdot \frac{D p_{1}}{D t} .
$$

It follows, after taking the limit $t \rightarrow 0_{+}$and using (2.4) again, that

$$
-\frac{1}{\rho_{*}} p_{L}^{\prime}-c_{*} u_{L}^{\prime}=\left(\frac{\partial u}{\partial t}\right)_{*}-\frac{u_{*}-c_{*}}{\rho_{*} c_{*}^{2}} \cdot\left(\frac{D p}{D t}\right)_{*} .
$$

By resolving the acoustic wave moving to the right, we get

$$
-\frac{1}{\rho_{*}} p_{R}^{\prime}+c_{*} u_{R}^{\prime}=\left(\frac{\partial u}{\partial t}\right)_{*}-\frac{u_{*}+c_{*}}{\rho_{*} c_{*}^{2}} \cdot\left(\frac{D p}{D t}\right)_{*} .
$$

We combine (6.5) and (6.6) to yield $(\partial u / \partial t)_{*}$ in $(6.1)$ and

$$
\frac{2}{\rho_{*} c_{*}} \cdot\left(\frac{D p}{D t}\right)_{*}=-\frac{1}{\rho_{*}} p_{L}^{\prime}-c_{*} u_{L}^{\prime}+\frac{1}{\rho_{*}} p_{R}^{\prime}-c_{*} u_{R}^{\prime} .
$$

Then using (2.1) and (2.4) again, we obtain $(\partial p / \partial t)_{*}$ as follows,

$$
\left(\frac{D u}{D t}\right)_{*}=\left(\frac{\partial u}{\partial t}\right)_{*}-\frac{u_{*}}{\rho_{*} c_{*}^{2}}\left(\frac{D p}{D t}\right)_{*}, \quad\left(\frac{\partial p}{\partial t}\right)_{*}=\left(\frac{D p}{D t}\right)_{*}+\rho_{*} u_{*}\left(\frac{D u}{D t}\right)_{*} .
$$

After getting $(\partial p / \partial t)_{*}$, we use the equation of state $p=p(\rho, S)$ to obtain $(\partial \rho / \partial t)_{*}$. We consider the case that $u_{*}>0$. Then we have

$$
\begin{aligned}
\left(\frac{\partial p}{\partial t}\right)_{*} & =c_{*}^{2}\left(\frac{\partial \rho}{\partial t}\right)_{*}+\frac{\partial p}{\partial S}\left(\rho_{*}, S_{*}\right) \cdot\left(\frac{\partial S}{\partial t}\right)_{*} \\
& =c_{*}^{2}\left(\frac{\partial \rho}{\partial t}\right)_{*}-u_{*} \frac{\partial p}{\partial S}\left(\rho_{*}, S_{*}\right) \cdot\left(\frac{\partial S}{\partial x}\right)_{*} \\
& =c_{*}^{2}\left(\frac{\partial \rho}{\partial t}\right)_{*}-u_{*} \frac{\partial p}{\partial S}\left(\rho_{L}, S_{L}\right) \cdot S_{L}^{\prime} \\
& =c_{*}^{2}\left(\frac{\partial \rho}{\partial t}\right)_{*}-u_{*}\left(p_{L}^{\prime}-c_{*}^{2} \rho_{L}^{\prime}\right) .
\end{aligned}
$$

This gives (6.2).

Remark 6.2. We can take the limit $U_{L}=U_{*}=U_{R}$ for the results in Section 5 to get Theorem 6.1. Another approach to prove Theorem 6.1 is to use a standard linearization method around the state $U_{*}$ with the rigorous justification. 


\section{Two Dimensional EXTENSION}

We use the Strang splitting [20, 4, Chapter 7] for the two dimensional compressible Euler system,

$$
\begin{gathered}
\rho_{t}+\nabla \cdot(\rho V)=0 \\
(\rho V)_{t}+\nabla \cdot(\rho V \otimes V+p)=0 \\
(\rho E)_{t}+\nabla \cdot(V(\rho E+p))=0,
\end{gathered}
$$

where, in addition to the thermodynamical flow variables $\rho, p$ and $e, V=(u, v)$ is the velocity and $E=\left(u^{2}+v^{2}\right) / 2+e$.

The Strang method splits (7.1) into two subsystems,

$$
\left\{\begin{array} { l } 
{ \frac { \partial \rho } { \partial t } + \frac { \partial ( \rho u ) } { \partial x } = 0 , } \\
{ \frac { \partial ( \rho u ) } { \partial t } + \frac { \partial ( \rho u ^ { 2 } + p ) } { \partial x } = 0 , } \\
{ \frac { \partial ( \rho v ) } { \partial t } + \frac { \partial ( \rho u v ) } { \partial x } = 0 , } \\
{ \frac { \partial ( \rho E ) } { \partial t } + \frac { \partial u ( \rho E + p ) } { \partial x } = 0 , }
\end{array} \quad \left\{\begin{array}{l}
\frac{\partial \rho}{\partial t}+\frac{\partial(\rho v)}{\partial y}=0 \\
\frac{\partial(\rho u)}{\partial t}+\frac{\partial(\rho u v)}{\partial y}=0 \\
\frac{\partial(\rho v)}{\partial t}+\frac{\partial\left(\rho v^{2}+p\right)}{\partial y}=0 \\
\frac{\partial(\rho E)}{\partial t}+\frac{\partial v(\rho E+p)}{\partial y}=0
\end{array}\right.\right.
$$

We denote by $\mathcal{L}_{x}(\Delta t), \mathcal{L}_{y}(\Delta t)$ the one dimensional evolution operator for one time step for the subsystems in (7.2) respectively. Then the evolution of one time step of the 2D Strang splitting algorithm is given by

$$
U^{n+1}=\mathcal{L}_{x}\left(\frac{\Delta t}{2}\right) \mathcal{L}_{y}(\Delta t) \mathcal{L}_{x}\left(\frac{\Delta t}{2}\right) U^{n}
$$

Therefore it suffices to consider the subsystem in the $x$-direction in (7.2). Then, in addition to the GRP resolution we already obtained for $\rho, u$ and $p$, we just need to provide the resolution for the velocity component $v$. Note that the component $v$ is transported with the speed $u$,

$$
\frac{\partial v}{\partial t}+u \frac{\partial v}{\partial x}=0
$$

Theorem 7.1. Assume that a rarefaction wave moves to the left and a shock wave moves to the right, and the line $x=0$ is located in the intermediate region, see Figure 2.1. Then

(i) If $u_{*} \geq 0$, the value $(\partial v / \partial t)_{*}$ is computed from the rarefaction wave (left hand) side as follows,

$$
\left(\frac{\partial v}{\partial t}\right)_{*}=-u_{*} \cdot \frac{\rho_{*}}{\rho_{L}} \cdot v_{L}^{\prime}
$$


(ii) If $u_{*}<0$, the value $(\partial v / \partial t)_{*}$ is computed from the shock wave (right hand) side, and

$$
\left(\frac{\partial v}{\partial t}\right)_{*}=-\frac{u_{*}\left(\sigma-u_{R}\right)}{\sigma-u_{*}} v_{R}^{\prime}
$$

Proof. In the case that $u_{*}>0$, the contact discontinuity moves to the right, and $v$ is continuous in the region to the left of this contact discontinuity. Since $v$ is constant along particle lines, if we take $x_{2}<x_{1}<0$ and let $v_{2}$ and $v_{1}$ be the corresponding values of $v$, we get $v_{i}=v\left(x_{i}(t), t\right)$, where $x_{i}(t)$ is the particle trajectory starting at $x_{i}, i=1,2$. The amount of mass $\Delta m(t)=\int_{x_{2}(t)}^{x_{1}(t)} \rho(x, t) d x$ between the two particles is also conserved, so that we get

$$
\frac{v_{2}-v_{1}}{\Delta m(0)}=\frac{v\left(x_{2}(t), t\right)-v\left(x_{1}(t), t\right)}{\Delta m(t)}
$$

Letting $x_{2}$ tend to zero, we obtain

$$
\left(\frac{\partial v}{\partial x}\right)_{*}=\frac{\rho_{*}}{\rho_{L}} \cdot v_{L}^{\prime}
$$

Then (7.5) follows by using Eq. (7.4).

For $u_{*}<0$, the contact discontinuity moves to the left. Then we need to compute $(\partial v / \partial t)_{*}$ from the right-hand side (shock side). Since $v$ is continuous across the shock $x=x(t)$, $v(x(t)-0, t)=v(x(t)+0, t)$, and the directional derivative of $v$ the shock trajectory $x=x(t)$ is also continuous. Hence we have

$$
\frac{\partial v(x(t)-0, t)}{\partial t}+\sigma \frac{\partial v(x(t)-0, t)}{\partial x}=\frac{\partial v(x(t)+0, t)}{\partial t}+\sigma \frac{\partial v(x(t)+0, t)}{\partial x} .
$$

Letting $t \rightarrow 0_{+}$, we have

$$
\frac{\partial v}{\partial x}(x(t)+0, t) \rightarrow v_{R}^{\prime}, \quad \frac{\partial v}{\partial t}(x(t)+0, t) \rightarrow-u_{R} v_{R}^{\prime},
$$

and

$$
\frac{\partial v(x(t)-0, t)}{\partial t} \rightarrow\left(\frac{\partial v}{\partial t}\right)_{*}, \quad \frac{\partial v(x(t)-0, t)}{\partial x}=-\frac{1}{u_{*}} \cdot\left(\frac{\partial v}{\partial t}\right)_{*} .
$$

Inserting them into (7.9) yields (7.6).

We remark at this point that although the velocity component $v$ is continuous across a rarefaction or a shock, the derivative of $v$ cannot be computed simply from one side as in the acoustic case due to the nonlinear effect. Indeed, Eqs. (7.5) and (7.6) correct the mistake in the original GRP scheme, see the formula (7.26) in [4, Page 247].

\section{IMPLEMENTATION OF THE GRP SCHEME}

In this section we describe the one dimensional implementation of the GRP scheme through the following four steps.

Step 1. Given piecewise initial data

$$
U^{n}(x)=U_{j}^{n}+\sigma_{j}^{n}\left(x-x_{j}\right), \quad x \in\left[x_{j-1 / 2}, x_{j+1 / 2}\right],
$$


we solve the associated Riemann problem for (1.1) ((7.2) for 2-D splitting) to define the Riemann solution

$$
U_{j+1 / 2}^{n}=R^{A}\left(0 ; U_{j}^{n}+\frac{\Delta x}{2} \sigma_{j}^{n}, U_{j+1}^{n}-\frac{\Delta x}{2} \sigma_{j+1}^{n}\right) .
$$

This is the same as the classical Godunov scheme [10], i.e, in the case of piecewise constant data $\sigma_{j}^{n} \equiv 0$.

Step 2. Determine $(\partial U / \partial t)_{j+1 / 2}^{n}$ according to Theorems 5.2, 5.4, 5.5 and 6.1 (in the acoustic case). All coefficients are summarized in Appendix A. Then calculate the numerical fluxes using Eq. (1.4).

Step 3. Evaluate the new cell averages $U_{j}^{n+1}$ using Eq. (1.3).

Step 4. Update the slopes $\sigma_{j}^{n+1}$ by the following procedure. Define

$$
\begin{gathered}
U_{j+1 / 2}^{n+1,-}=U_{j+1 / 2}^{n}+\Delta t\left(\frac{\partial U}{\partial t}\right)_{j+1 / 2}^{n}, \\
\sigma_{j}^{n+1}=\frac{1}{\Delta x}(\Delta U)_{j}^{n+1,-}:=\frac{1}{\Delta x}\left(U_{j+1 / 2}^{n+1,-}-U_{j-1 / 2}^{n+1,-}\right) .
\end{gathered}
$$

In order to suppress local oscillations near discontinuities, we apply to $\sigma_{j}^{n+1}$ a monotonicity algorithm-slope limiters, see [1,22].

Remark 8.1. In comparison with the classical Godunov scheme (Step 1), we only need to add Step 2 giving $(\partial U / \partial t)_{j+1 / 2}^{n}$. This is accomplished at most by solving two linear algebraic equations at each grid point. In most cases (sonic, acoustic etc.), the computation of $(\partial U / \partial t)_{j+1 / 2}^{n}$ is very simple, see Theorems 5.4 and 6.1 .

Remark 8.2. If the difference of $U_{j+1 / 2,-}^{n}$ and $U_{j+1 / 2,+}^{n}$ is relatively small, the acoustic case can be used, see Theorem 6.1. The resulting scheme is called the $E_{1}$ - scheme. In contrast, if the general case in Section 5 is used, we label the resulting scheme the $E_{\infty}$-scheme.

\section{Numerical EXAMPles}

We choose several one-dimensional and two-dimensional examples to illustrate the performance of our scheme. They are one-dimensional Riemann problems, the interaction of one-dimensional waves, and three two dimensional Riemann problems. All of them were often used as test problems to check numerical schemes.

9.1. One dimensional examples. We choose five well-understood one-dimensional examples to test our scheme.

(a) Sod problem. As commonly used, our first example is the shock tube problem by Sod [19]. The gas is initially at rest with $\rho=1, p=1$ for $0 \leq x \leq 50$ and $\rho=0.125, p=0.1$ for $50<x \leq 100$. Numerical results are shown at time $\mathrm{t}=15.0$ in Figure 9.1. The solid lines represent the exact solutions, while the dots stand for numerical solutions. We can see that our scheme does very well in the smooth region, and is comparable at discontinuities with other schemes.

(b) Nearly stationary shock. Initially, $\rho=4.0, p=4 / 3, u=-0.3$ for $0 \leq x<20$; and $\rho=1.0, p=10^{-6}$ and $u=-1.3$ for $20<x \leq 100$. The polytropic index is taken to be $\gamma=5 / 3$. The result is shown at time $T=2000$ in Figure 9.2. This example involves a 

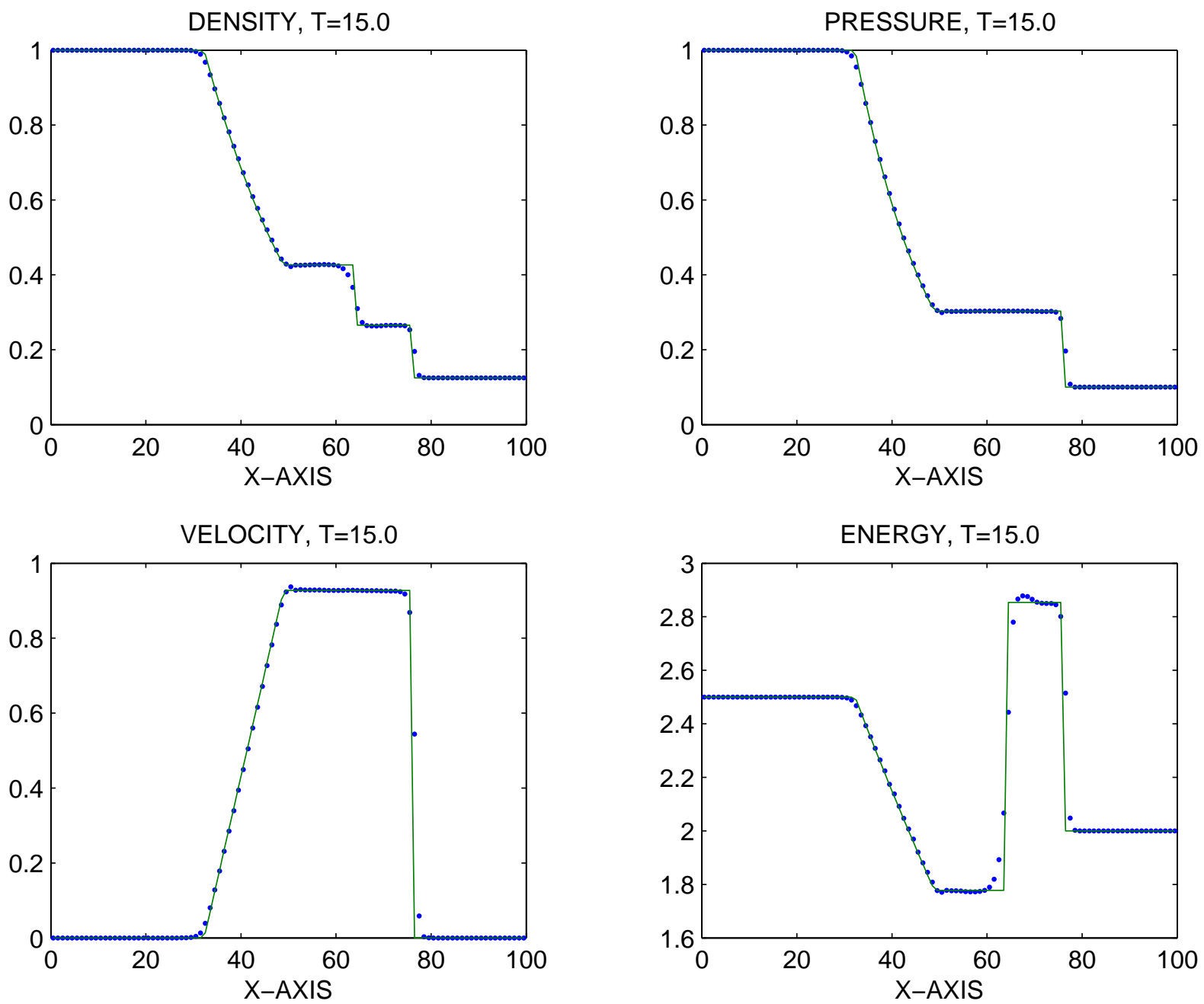

Figure 9.1. (a) Numerical results for Sod's problem: 100 grid points are used.

very strong nearly stationary shock, whose exact speed is $3.4052 \times 10^{-2}$. This is an almost infinite shock in the sense that the density ratio is close to its maximum. The "wavelike" behavior can be smoothed out by enhancing the dissipative mechanism, as pointed out [1].

(c) Shock and contact interaction. This example was proposed in [4, Section 6.2.1]. The initial data are given at time $t=-10,(\rho, u, p)=(2.8182,1.6064,5.0)$ for $x<-24.90$, $(\rho, u, p)=(1,0,1)$ for $-24.90 \leq x<0$ and $(\rho, u, p)=(0.3,0,1.0)$ for $x \geq 0$. A shock emanates from $(-24.90,-10)$ and propagates to the right. It interacts at time $t=0$ with the contact discontinuity emanating from $(0,-10)$. Then a rarefaction wave, a contact discontinuity and a shock are produced at $(0,0)$. Figure 9.3 displays numerical solutions within $[-20,90]$. We see the solution is quite accurate (of course the contact discontinuity is obviously smeared as in most second order schemes).

(d) Interacting blast wave problem [23]. The gas is at rest and ideal with $\gamma=1.4$, and the density is everywhere unit. The pressure is $p=1000$ for $0 \leq x<10$ and $p=100$ for $90<x \leq 100$, while it is only $p=0.01$ in $10<x<90$. Reflecting boundary conditions are applied at both ends. Numerical solutions are shown in Figures 9.4 and 9.5. In both Figures 

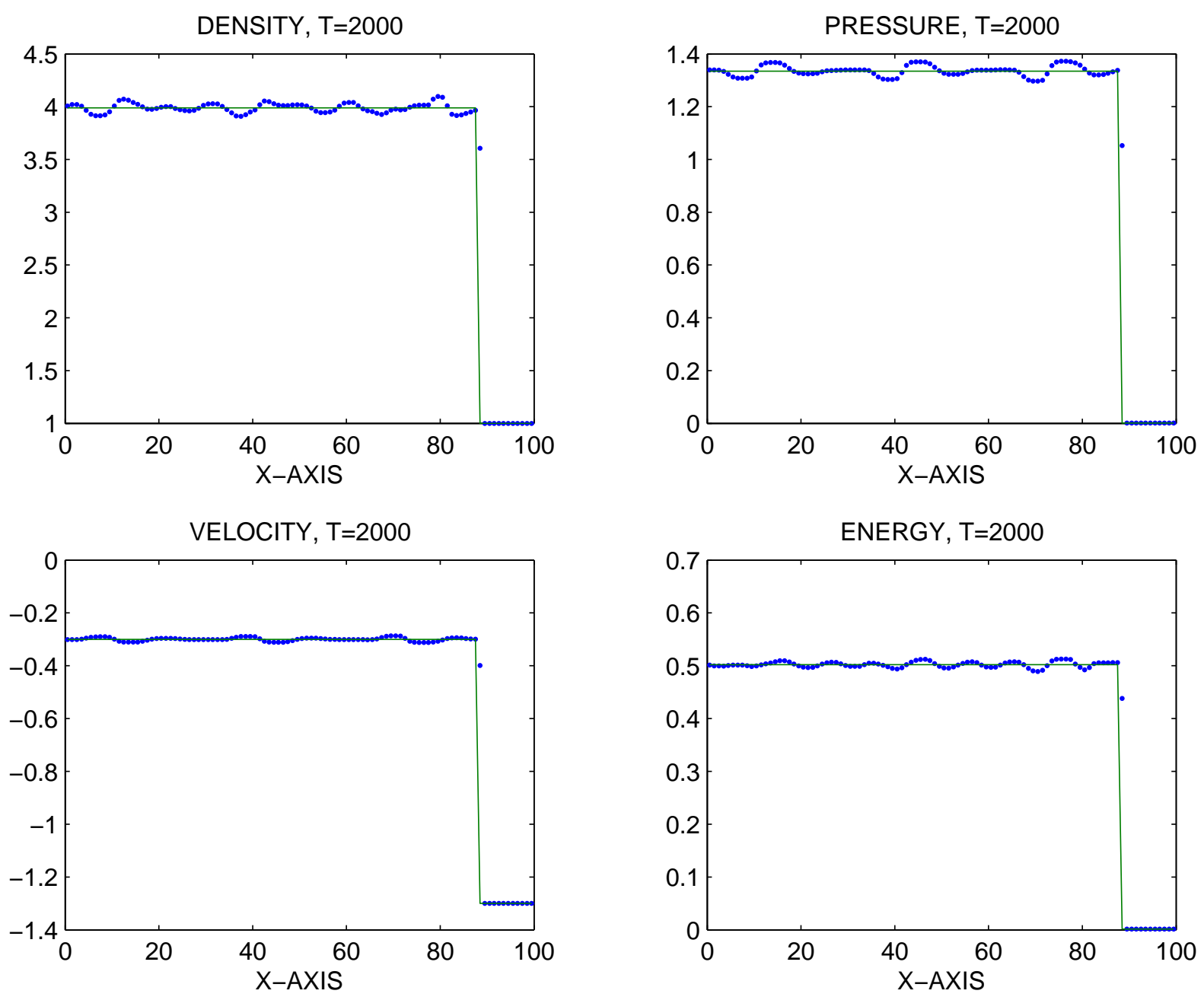

FIGURE 9.2. (b) Numerical results for a very strong nearly stationary shock: 100 grid points are used.

the solid lines are obtained with 3200 grid points, while we use 200 grid points for the dots in Figure 9.4, and 800 grid points is used for the dots in Figure 9.5.

(e) Low density and internal energy Riemann problem $[8,16]$. The initial data is given with $(\rho, u, p)=(1,-2,0.4)$ for $0 \leq x<50$ and $(\rho, u, p)=(1,2,0.4)$ for $50 \leq x \leq 100$. The numerical result is shown in Figure 9.6. The solid lines are obtained with the exact Riemann solvers in [21]. The dotted lines are obtained with 100 points. This example shows that the GRP scheme can preserve the positivity of the density, pressure and energy.

9.2. Two-dimensional Riemann problems. We choose three two-dimensional Riemann problems as our examples. The two-dimensional Riemann problems were proposed by $\mathrm{T}$. Zhang and Y. Zheng [24], then followed by many numerical simulations [17, 16, 6, 11] etc. Systematic treatments can be found in [14, 25]. The flow patterns are quite complex, including the Mach reflection, rolling up of slip lines, formation of shocks and much more. Nowadays the two-dimensional Riemann problems have been useful tests for checking the 

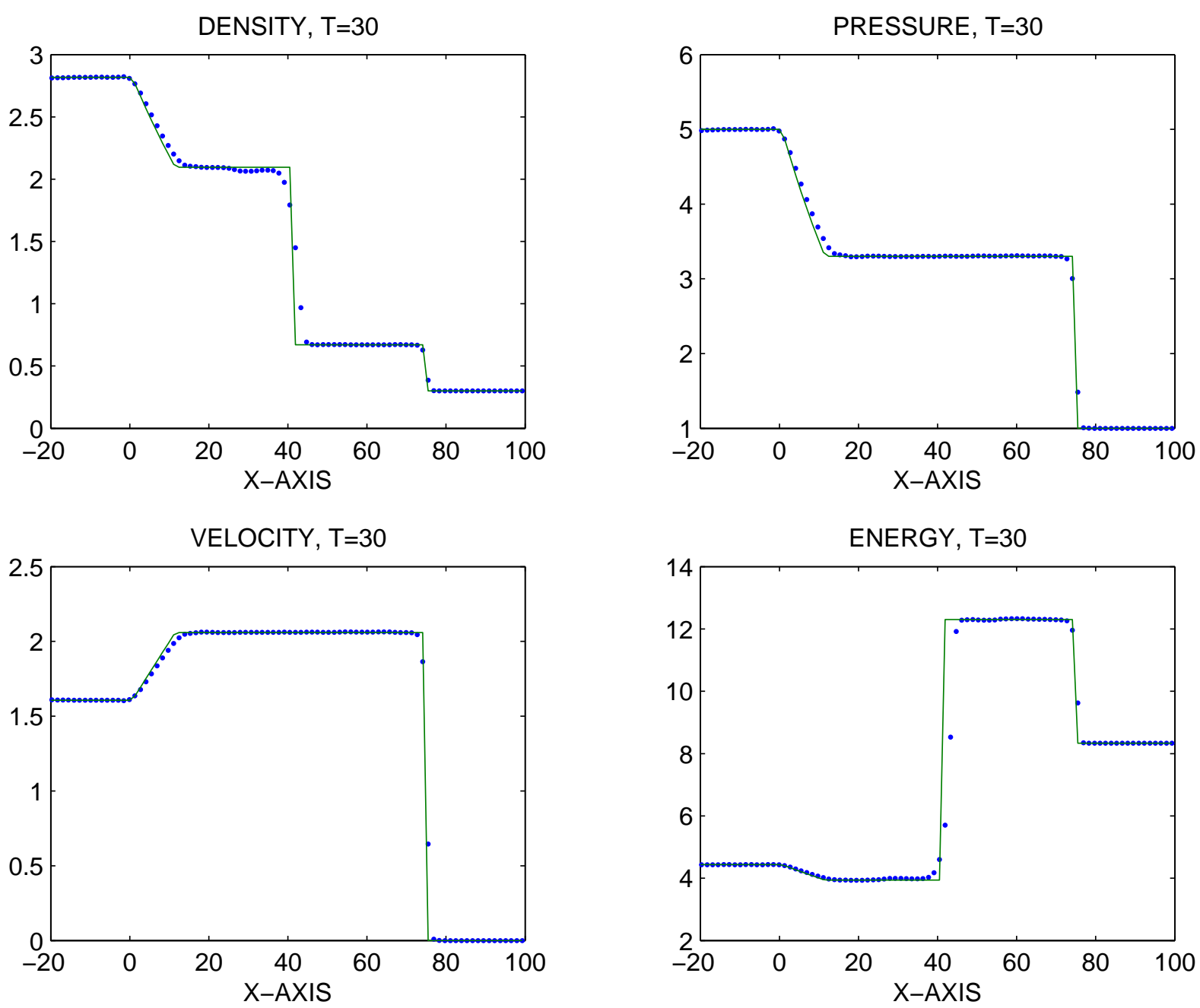

FiguRE 9.3. (c) Numerical results for shock and contact interaction: 100 grid points are used.

accuracy of numerical schemes in several dimensions. We present three examples with contour curves of density in all three examples. The initial data for each example consists of four constant states in the four quadrants. Furthermore, the initial data is designed so that only one elementary wave, a shock, a rarefaction wave or a contact discontinuity, emanates from each initial discontinuity along the coordinate axes. We use the notation $\left(\rho_{i}, u_{i}, v_{i}, p_{i}\right)$ to express the constant state in the $i$-th quadrant, $i=1,2,3,4$.

(f) The interaction of vortex sheets and the formation of spiral. The Riemann initial data is chosen to be $\rho_{1}=0.5, u_{1}=0.5, v_{1}=-0.5, p_{1}=5 ; \rho_{2}=1.0, u_{2}=0.5$, $v_{2}=0.5, p_{2}=5 ; \rho_{3}=2.0, u_{3}=-0.5, v_{3}=0.5, p_{3}=5 ;$ and $\rho_{4}=1.5, u_{4}=-0.5, v_{4}=-0.5$, $p_{4}=5$. Initially four vortex sheets are supported on the $x$ and $y$ axes with the same sign, but they have different measures. They interact and form a spiral, as shown in Figure 9.7. In the center of the spiral, the density is very low. Compared to $[14,6,11,16,17]$, Figure 9.7 displays a more accurate result. 

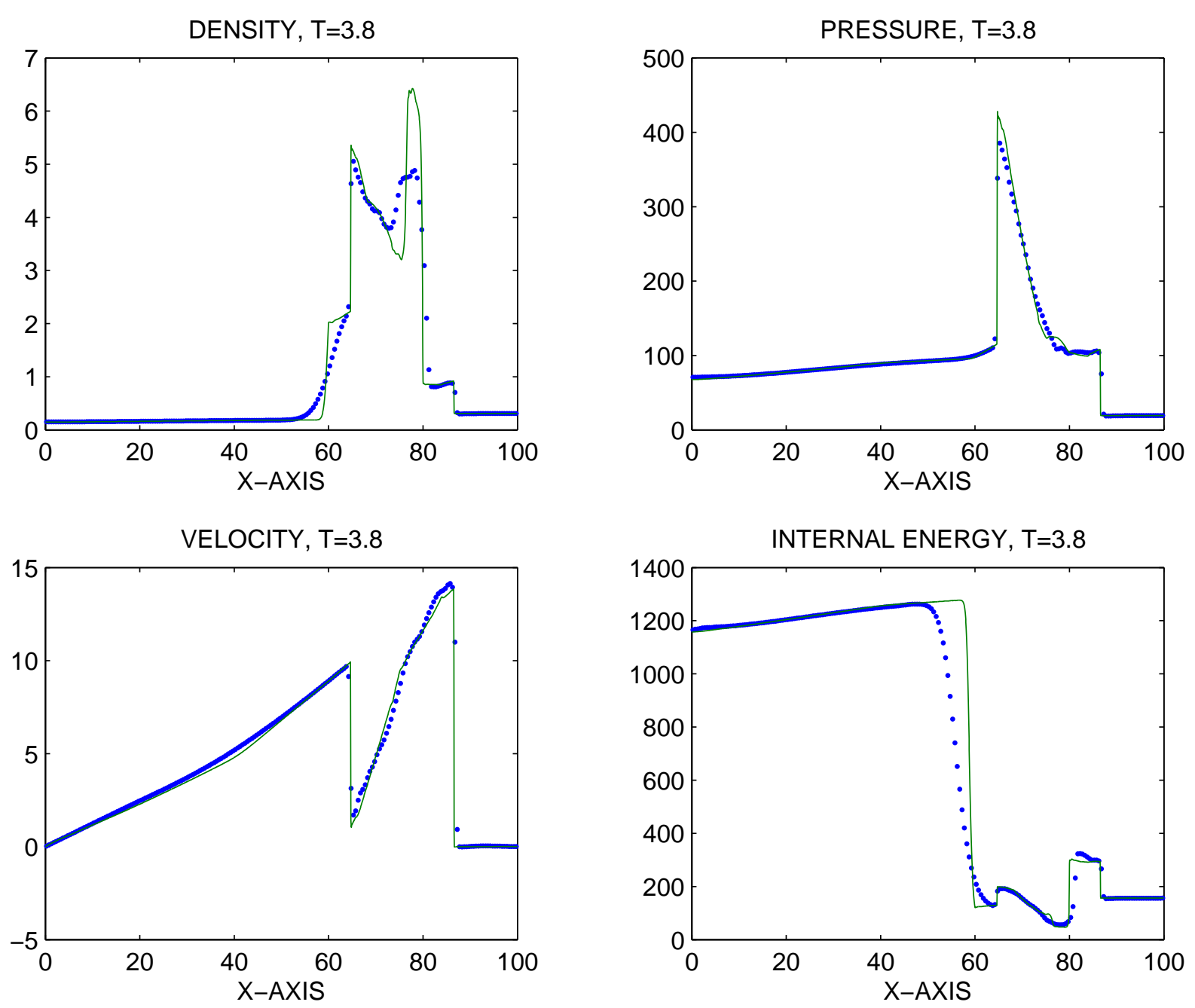

FiguRE 9.4. (d) Numerical results for the interacting blast wave problem: 200 grid points are used.

(g) Interaction of shocks. This is the 2-D Riemann problem for interacting shocks. It was Configuration $\mathrm{C}$ in [14, Page 244]. See also $[6,11,16,17]$. The initial data is $\rho_{1}=1.5$, $u_{1}=0.0, v_{1}=0.0, p_{1}=1.5 ; \rho_{2}=0.5323, u_{2}=1.206, v_{2}=0.0, p_{2}=0.3 ; \rho_{3}=0.138$, $u_{3}=1.206, v_{3}=1.206, p_{3}=0.029 ;$ and $\rho_{4}=0.5323, u_{4}=0.0, v_{4}=1.206, p_{4}=0.3$. Initially a single planar shock emanates from each coordinate axis. The four shock interact as time evolves, and a very complicated wave pattern emerges. It includes the triple points, Mach stems and contact discontinuities etc. The numerical result is displayed in Figure 9.8 and reflects conspicuous phenomenon in the oblique shock experiments.

(h) The formation of shocks in the interaction of planar rarefactions. We check the interaction of four 2-D planar rarefaction waves, see Figure 9.9. The Riemann initial data are $\rho_{1}=1.0, u_{1}=0.0, v_{1}=0.0, p_{1}=1.0 ; \rho_{2}=0.5197, u_{2}=-0.7259, v_{2}=0.0, p_{2}=0.4$; $\rho_{3}=1.0, u_{3}=-0.7259, v_{3}=-0.7259, p_{3}=1.0 ;$ and $\rho_{4}=0.5197, u_{4}=0.0, v_{4}=-0.7259$, $p_{4}=0.4$. Initially, there are four planar rarefaction wave emanating from the coordinate axis, respectively and they interact. We observe that two symmetric compressive waves in 

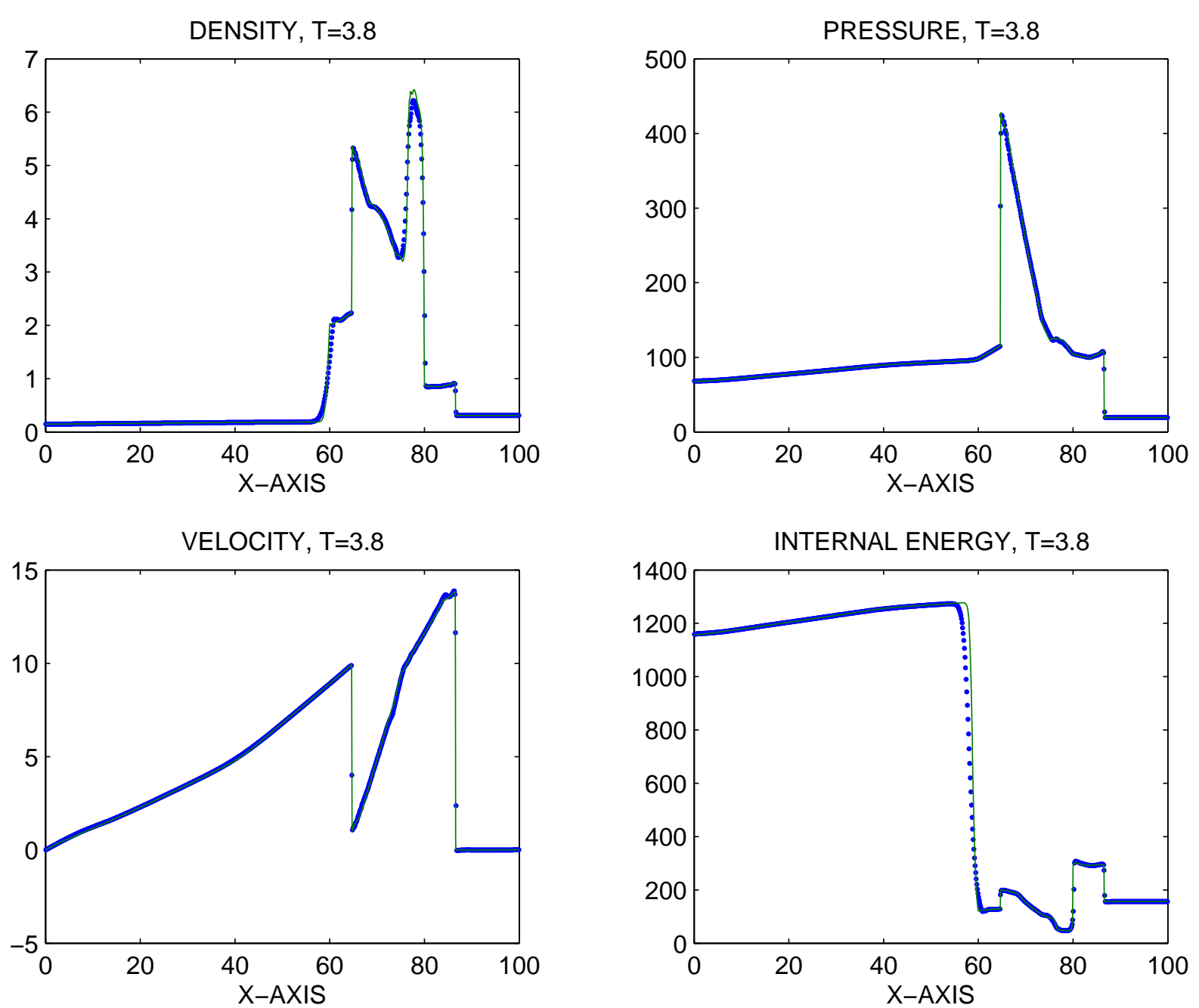

FiguRE 9.5. (d) Numerical results for the interacting blast wave problem: 800 grid points are used.

the domain where the rarefaction waves interact. The numerical results are consistent with those in $[14,17,16,6,11]$. This is a typical two dimensional phenomenon, which never emerges in the interaction of rarefaction waves in one dimension.

\section{Appendix A. Useful Coefficients for the GRP scheme}

A.1. The coefficients in Theorem $\mathbf{5 . 1}$ for all cases. In Table II, we collect for all cases the coefficients of the system of the linear algebraic equations in Theorem 5.1 for the polytropic gases. Here we assume that the $t$-axis (cell interface) is located inside the intermediate region. In this table, the 1 -shock (resp. 3-shock) refers to as the shock associated with the $u-c$ characteristic family (resp. $u+c$ ). Analogously for the 1-rarefaction wave and the 3 -rarefaction wave. 

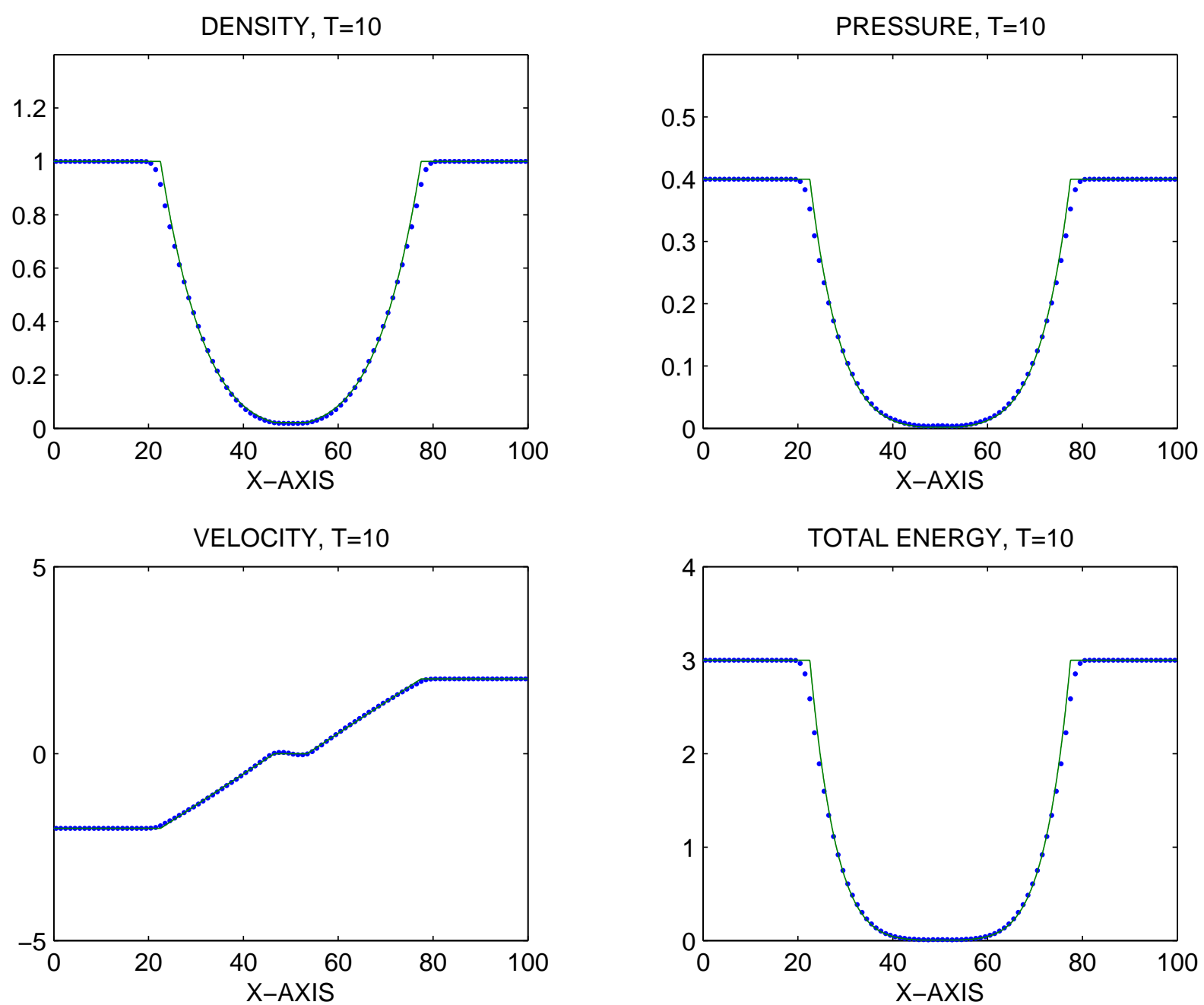

FiguRE 9.6. (e) Numerical results for the low density and energy problem: 100 grid points are used.

TABLE II

\begin{tabular}{ll}
\hline Two rarefaction waves & $\left(a_{L}, b_{L}\right)=\left(a_{L}^{\text {rare }}, b_{L}^{\text {rare }}\right), d_{L}=d_{L}^{\text {rare }}$ \\
& $\left(a_{R}, b_{R}\right)=\left(a_{R}^{\text {rare }}, b_{R}^{\text {rare }}\right), d_{R}=d_{R}^{\text {rare }}$ \\
\hline Two shocks & $\left(a_{L}, b_{L}\right)=\left(a_{L}^{\text {shock }}, b_{L}^{\text {shock }}\right), d_{L}=d_{L}^{\text {shock }}$ \\
& $\left(a_{R}, b_{R}\right)=\left(a_{R}^{\text {shock}}, b_{R}^{\text {shock }}\right), d_{R}=d_{R}^{\text {shock }}$ \\
\hline 1-shock and 3-rarefaction wave & $\left(a_{L}, b_{L}\right)=\left(a_{L}^{\text {shock }}, b_{L}^{\text {shock }}\right), d_{L}=d_{L}^{\text {shock }}$ \\
& $\left(a_{R}, b_{R}\right)=\left(a_{R}^{\text {rare }}, b_{R}^{\text {rare }}\right), d_{R}=d_{R}^{\text {rare }}$ \\
\hline 1-rarefaction wave and 3-shock & $\left(a_{L}, b_{L}\right)=\left(a_{L}^{\text {rare }}, b_{L}^{\text {rare }}\right), d_{L}=d_{L}^{\text {rare }}$ \\
& $\left(a_{R}, b_{R}\right)=\left(a_{R}^{\text {shock }}, b_{R}^{\text {shock }}\right), d_{R}=d_{R}^{\text {shock }}$ \\
\hline
\end{tabular}




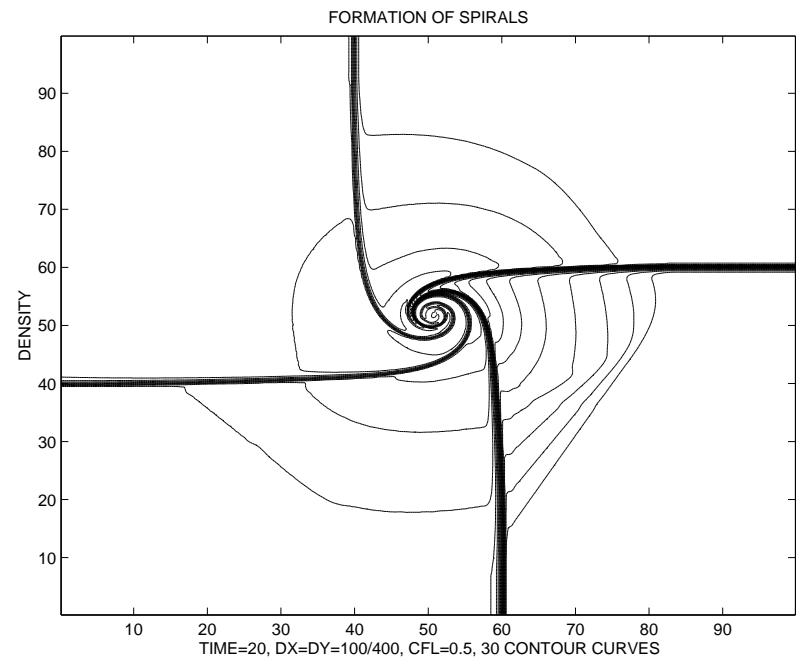

FiguRE 9.7. (f) Numerical results for interaction of four contact discontinuities.

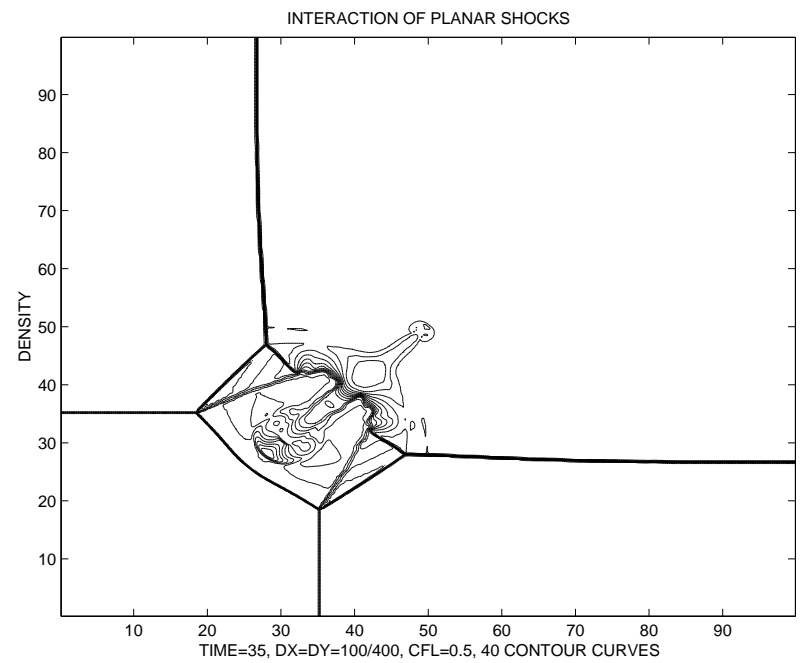

FiguRE 9.8. (g) Numerical results for interaction of four planar shocks.

The coefficients for rarefaction waves are given by (A.1)

$$
\begin{gathered}
\left(a_{L}^{\text {rare }}, b_{L}^{\text {rare }}\right)=\left(1, \frac{1}{\rho_{1 *} c_{1 *}}\right), \quad\left(a_{R}^{\text {rare }}, b_{R}^{\text {rare }}\right)=\left(1,-\frac{1}{\rho_{2 *} c_{2 *}}\right) \\
d_{L}^{\text {rare }}=\left[\frac{1+\mu^{2}}{1+2 \mu^{2}}\left(\frac{c_{1 *}}{c_{L}}\right)^{1 /\left(2 \mu^{2}\right)}+\frac{\mu^{2}}{1+2 \mu^{2}}\left(\frac{c_{1 *}}{c_{L}}\right)^{\left(1+\mu^{2}\right) / \mu^{2}}\right] T_{L} S_{L}^{\prime}-c_{L}\left(\frac{c_{1 *}}{c_{L}}\right)^{1 /\left(2 \mu^{2}\right)} \psi_{L}^{\prime} . \\
d_{R}^{\text {rare }}=\left[\frac{1+\mu^{2}}{1+2 \mu^{2}}\left(\frac{c_{2 *}}{c_{R}}\right)^{1 /\left(2 \mu^{2}\right)}+\frac{\mu^{2}}{1+2 \mu^{2}}\left(\frac{c_{2 *}}{c_{R}}\right)^{\left(1+\mu^{2}\right) / \mu^{2}}\right] T_{R} S_{R}^{\prime}+c_{R}\left(\frac{c_{2 *}}{c_{R}}\right)^{1 /\left(2 \mu^{2}\right)} \phi_{R}^{\prime} .
\end{gathered}
$$




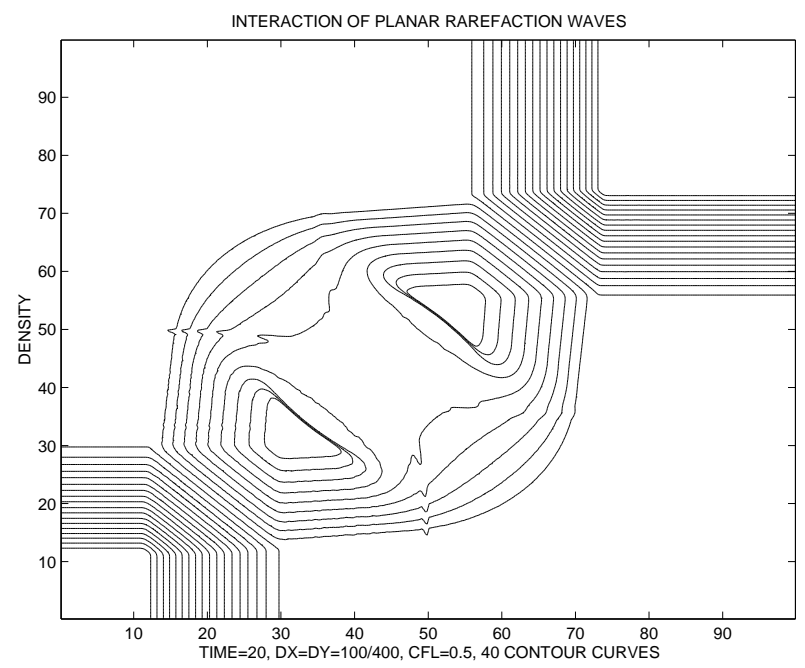

Figure 9.9. (h) Numerical results for interaction of four planar rarefaction waves.

The coefficients for shock waves are given by (A.2)

$$
\begin{gathered}
a_{L}^{\text {shock }}=1-\rho_{1 *}\left(\sigma_{L}-u_{*}\right) H_{1}\left(p_{*} ; p_{L}, \rho_{L}\right), \quad b_{L}^{\text {shock }}=-\frac{1}{\rho_{1 *} c_{1 *}^{2}}\left(\sigma_{L}-u_{*}\right)+H_{1}\left(p_{*} ; p_{L}, \rho_{L}\right), \\
d_{L}^{\text {shock }}=L_{p}^{L} p_{L}^{\prime}+L_{u}^{L} u_{L}^{\prime}+L_{\rho}^{L} \rho_{L}^{\prime}, \\
a_{R}^{\text {shock }}=1+\rho_{2 *}\left(\sigma_{R}-u_{*}\right) H_{1}\left(p_{*} ; p_{R}, \rho_{R}\right), \quad b_{R}^{\text {shock }}=-\left[\frac{1}{\rho_{2 *} c_{2 *}^{2}}\left(\sigma_{R}-u_{*}\right)+H_{1}\left(p_{*} ; p_{R}, \rho_{R}\right)\right], \\
d_{R}^{\text {shock }}=L_{p}^{R} p_{R}^{\prime}+L_{u}^{R} u_{R}^{\prime}+L_{\rho}^{R} \rho_{R}^{\prime},
\end{gathered}
$$

where all quantities involved are

(A.3)

$$
\begin{array}{ll}
L_{p}^{L}=-\frac{1}{\rho_{L}}-\left(\sigma_{L}-u_{L}\right) H_{2}\left(p_{*} ; p_{L}, \rho_{L}\right), & L_{u}^{L}=\sigma_{L}-u_{L}+\rho_{L} c_{L}^{2} H_{2}\left(p_{*} ; p_{L}, \rho_{L}\right)+\rho_{L} H_{3}\left(p_{*} ; p_{L}, \rho_{L}\right), \\
L_{\rho}^{L}=-\left(\sigma_{L}-u_{L}\right) H_{3}\left(p_{*} ; p_{L}, \rho_{L}\right), & \sigma_{L}=\frac{\rho_{1 *} u_{*}-\rho_{L} u_{L}}{\rho_{1 *}-\rho_{L}}, \\
L_{p}^{R}=-\frac{1}{\rho_{R}}+\left(\sigma_{R}-u_{R}\right) H_{2}\left(p_{*} ; p_{R}, \rho_{R}\right), & L_{u}^{R}=\sigma_{R}-u_{R}-\rho_{R} c_{R}^{2} H_{2}\left(p_{*} ; p_{R}, \rho_{R}\right)-\rho_{R} H_{3}\left(p_{*} ; p_{R}, \rho_{R}\right), \\
L_{\rho}^{R}=\left(\sigma_{R}-u_{R}\right) H_{3}\left(p_{*} ; p_{R}, \rho_{R}\right), & \sigma_{R}=\frac{\rho_{2 *} u_{*}-\rho_{R} u_{R}}{\rho_{2 *}-\rho_{R}},
\end{array}
$$


and $\left(\operatorname{denote}(\bar{p}, \bar{\rho})=\left(p_{L}, \rho_{L}\right)\right.$ or $\left.(\bar{p}, \bar{\rho})=\left(p_{R}, \rho_{R}\right)\right)$,

$$
H_{1}(p ; \bar{p}, \bar{\rho})=\frac{1}{2} \sqrt{\frac{1-\mu^{2}}{\bar{\rho}\left(p+\mu^{2} \bar{p}\right)}} \cdot \frac{p+\left(1+2 \mu^{2}\right) \bar{p}}{p+\mu^{2} \bar{p}},
$$

$$
\begin{gathered}
H_{2}(p ; \bar{p}, \bar{\rho})=-\frac{1}{2} \sqrt{\frac{1-\mu^{2}}{\bar{\rho}\left(p+\mu^{2} \bar{p}\right)}} \cdot \frac{\left(2+\mu^{2}\right) p+\mu^{2} \bar{p}}{p+\mu^{2} \bar{p}} \\
H_{3}(p ; \bar{p}, \bar{\rho})=-\frac{p-\bar{p}}{2 \bar{\rho}} \sqrt{\frac{1-\mu^{2}}{\bar{\rho}\left(p+\mu^{2} \bar{p}\right)}}
\end{gathered}
$$

A.2. Sonic case. When the $t$-axis is located inside the rarefaction waves associated with $u+c$. Then we have

$$
\left(\frac{\partial u}{\partial t}\right)_{*}=d_{R}^{r a r e}, \quad\left(\frac{\partial p}{\partial t}\right)_{*}=\rho_{*} u_{*} d_{R}^{r a r e}
$$

where $d_{R}^{\text {rare }}$ is given in (A.1).

\section{ACKNOWLEDGEMENT}

We would like to thank Professors J. Falcovitz, M. Lukacova and T. Zhang for their interest and discussion. Jiequan Li's research is supported by the Fellowship of Alexander von Humboldt, the grant NSF of China with No. 10301022, the Natural Science Foundation of Beijing, Fok Ying Tong Education Foundation and and the Key Program from Beijing Educational Commission with no. KZ200510028018..

\section{REFERENCES}

[1] M. Ben-Artzi and J. Falcovitz, A second-order Godunov-type scheme for compressible fluid dynamics, J. Comput. Phys., 55 (1984), no. 1, 1-32.

[2] M. Ben-Artzi and J. Falcovitz, An upwind second-order scheme for compressible duct flows, SIAM J. Sci. Statist. Comput., 7 (1986), no. 3, 744-768.

[3] M. Ben-Artzi, The generalized Riemann problem for reactive flows, J. Comput. Phys., 81 (1989), no. 1, $70-101$.

[4] M. Ben-Artzi and J. Falcovitz, Generalized Riemann problems in computational gas dynamics, Cambridge University Press, 2003.

[5] A. Bourgeade, P. LeFloch and P. -A. Raviart, An asymptotic expansion for the solution of the generalized Riemann problem. II. Application to the equations of gas dynamics Ann. Inst. H. Poincar Anal. Non Linaire, 6 (1989), no. 6, 437-480.

[6] T. Chang, G. -Q. Chen and S. Yang, On the 2-D Riemann problem for the compressible Euler equations I: Interaction of shocks and rarefaction waves. Discrete Contin. Dyn. Syst. 1, No.4, 555-584 (1995). II: Interaction of contact discontinuities. 6, No.2, 419-430 (2000).

[7] R. Courant and K. O. Friedrichs, Supersonic flow and shock waves, Interscience, New York, 1948.

[8] B. Einfeldt, C. D. Munz, P. L. Roe and B. Sjogreen, On Godunov-type methods near low densities. J. Comput. Phys. 92, No.2, 273-295 (1991).

[9] E. Godlewski and P.-A. Raviart, Numerical approximation of hyperbolic systems of conservation laws, Applied Mathematical Sciences 118, Springer, 1996.

[10] S. K. Godunov, A finite difference method for the numerical computation and disontinuous solutions of te equations of fluid dynamics, Mat. Sb. 47 (1959), 271-295. 
[11] A. Kurganov, E. Tadmor, Solution of two-dimensional Riemann problems for gas dynamics without Riemann problem solvers. Numer. Methods Partial Differ. Equations 18, No.5, 584-608 (2002).

[12] Ph. LeFloch and P. -A. Raviart, An asymptotic expansion for the solution of the generalized Riemann problem. I. General theory, Ann. Inst. H. Poincar é Anal. Non Liné aire, 5 (1988), no. 2, 179-207.

[13] J. Li and G. Chen, The generalized Riemann problem method for the shallow water equations with bottom topography, to appear in International Jouranl for Numerical Methods in Engineering, 2005.

[14] J. Li, T. Zhang and S. Yang, The two-dimensional Riemann problem in gas dynamics. Pitman Monographs and Surveys in Pure and Applied Mathematics. 98. Harlow: Addison Wesley Longman. (1998).

[15] T. T. Li, Global classical solutions for quasilinear hyperbolic systems. Research in Applied Mathematics. Chichester: Wiley. Paris: Masson, (1994).

[16] X.-D. Liu and P. D. Lax, Solution of two-dimensional Riemann problems of gas dynamics by positive schemes. SIAM J. Sci. Comput. 19, No.2, 319-340 (1998).

[17] C. W. Schulz-Rinne, J. P. Collins, and H. M. Glaz, Numerical solution of the Riemann problem for two-dimensional gas dynamics. SIAM J. Sci. Comput. 14, No.6, 1394-1414 (1993).

[18] J. Smoller, Shock waves and reaction-diffusion equations. 2nd ed. Grundlehren der Mathematischen Wissenschaften. 258. New York: Springer-Verlag, xxii, (1994).

[19] G. A. Sod, A survey of several finite difference methods for systems of nonlinear hyperbolic conservation laws. J. Comput. Phys., 27, 1-31 (1978).

[20] G. Strang, Accurate partial difference methods.I: Linear Cauchy problems, Arch. Ration. Mech. Anal. 12, 392-402 (1963); II: Non-linear problems, Numer. Math. 6, 37-46 (1964).

[21] E. F. Toro, Riemann solvers and numerical methods for fluid dynamics: A practical introducition, Springer, 1997.

[22] B. van Leer, Towards the ultimate conservative difference scheme, V. J. Comp. Phys., 32, 101-136(1979).

[23] P. Woodward, and P. Colella, The numerical simulation of two-dimensional fluid flow with strong shocks. J. Comput. Phys. 54, 115-173 (1984).

[24] T. Zhang and Y. Zheng, Conjecture on the structure of solutions of the Riemann problem for twodimensional gas dynamics systems. SIAM J. Math. Anal. 21, No.3, 593-630 (1990).

[25] Y. Zheng, Systems of conservation laws. Two-dimensional Riemann problems. Progress in Nonlinear Differential Equations and their Applications. 38. Boston, MA: Birkhauser. (2001).

Department of Mathematics, The Hebrew University of Jerusalem, 91904, Israel

Department of Mathematics, Capital Normal University, 100037, Beijing, P.R.China

Institute for Analysis and Numerics, Otto-von-Guericke University Magdeburg, D-39106, GERMANY

\author{
Matania Ben-Artzi: mbartzi@math.huji.ac.il \\ E-mail address: Jiequan Li: jiequan@mail.cnu.edu.cn \\ Gerald Warnecke: Gerald.Warnecke@Mathematik.Uni-Magdeburg.DE
}

